Applied Mathematics Reviews, World Sci. Pub. Co., vol. 1, (2000), 491-536

\title{
DYNAMICAL SYSTEMS AND DISCRETE METHODS FOR SOLVING NONLINEAR ILL-POSED PROBLEMS
}

\author{
RUBEN G. AIRAPETYAN AND ALEXANDER G. RAMM
}

\section{Contents}

1. Introduction

2. Continuous methods for well posed problems

3. Discretization theorems for well-posed problems

4. Nonlinear integral inequality

5. Regularization procedure for ill-posed problems

6. Discretization theorem for ill-posed problems

7. Regularized continuous methods for monotone operators

8. Regularized discrete methods for monotone operators

\section{INTRODUCTION}

The theme of this chapter is solving of nonlinear operator equation

$$
F(z)=0, \quad F: H \rightarrow H,
$$

by establishing a relation between the limiting behavior for large times of the trajectories of dynamical systems in $H$ and the solutions to equation (1.1) in a real Hilbert space $H$. We consider a real Hilbert space for the sake of simplicity, equation (1.1) in a complex Hilbert space can be treated similarly, and a part of results can be generalized to a Banach space.

A standard approach to numerical solution of equation (1.1) consists of using one of the numerous iterative methods. These methods are also very useful for a theoretical investigation of problem (1.1). For some operators $F$ they allow one to establish existence of a solution to problem (1.1), or existence and uniqueness theorems.

In the iterative methods one chooses some initial approximation $z_{0}$ and defines a sequence of points $\left\{z_{n}\right\}_{n=0,1,2, \ldots}$ having as a $\operatorname{limit}_{n \rightarrow \infty} \lim _{n \rightarrow \infty}$ one of the solutions of equation (1.1). The choice of an initial point $z_{0}$ is very important especially for problems solution to which is not unique. Usually the choice of $z_{0}$ determines to what solution the iterative sequence converges.

The simplest iterative method is the method of simple iteration defined as follows:

$$
z_{n}=z_{n-1}-F\left(z_{n-1}\right), \quad n=1,2, \ldots
$$

$z_{0}$ is given.

In the well-known Newton's method ([14, 18, 19]) one constructs a sequence $\left\{z_{n}\right\}$ by the following formula:

$$
z_{n}=z_{n-1}-\left[F^{\prime}\left(z_{n-1}\right)\right]^{-1} F\left(z_{n-1}\right), \quad n=1,2, \ldots .
$$


Here $F^{\prime}(h)$ is the Fréchet derivative of the operator $F$, defined at a point $h \in H$ as a linear operator from $H$ to $H$ such that:

$$
F(h+\xi)-F(h)=F^{\prime}(h) \xi+o(\|\xi\|) .
$$

Thus, an important condition for applicability of Newton's method is invertibility of the Fréchet derivative operator $F^{\prime}$. The important advantage of Newton's method is the quadratic convergence to the solution in a neighborhood of this solution:

$$
\left\|z_{n+1}-z_{n}\right\| \leq \mathrm{const}\left\|z_{n}-z_{n-1}\right\|^{2} .
$$

In many cases when Newton's method diverges one uses the damped Newton's method:

$$
z_{n}=z_{n-1}-\omega_{n}\left[F^{\prime}\left(z_{n-1}\right)\right]^{-1} F\left(z_{n-1}\right), \quad n=1,2, \ldots,
$$

where $\omega_{n}$ is an appropriately chosen sequence of positive numbers.

In the gradient method one constructs an iterative sequence using the formula:

$$
z_{n}=z_{n-1}-\left[F^{\prime}\left(z_{n-1}\right)\right]^{*} F\left(z_{n-1}\right), \quad n=1,2, \ldots .
$$

The iterative methods mentioned above are widely known representatives of a large family of iterative methods. A detailed description of many iterative methods one can find in [19]. See also an approach to construction of iterative process for solving nonlinear equation proposed in $[20,21]$.

The applicability of iterative methods is established by various convergence theorems (see $[14,18,19]$ ), which specify assumptions on the operator $F$ which guarantee convergence of the iterative sequence to a solution of equation (1.1). The proofs of convergence theorems for iterative methods are usually based on the contraction mapping principle.

Another approach to solving problem (1.1) is based on a construction of a dynamical system with the trajectory starting from an initial approximation point $z_{0}$ and having a solution to problem (1.1) as a limiting point.

In [12] R. Courant proposed the dynamical system, which is a continuous analog of the gradient method, in the problem of minimization of some functionals. In [16] M.K. Gavurin proposed continuous Newton's method and established the corresponding convergence theorem. In continuous Newton's method one considers the following Cauchy problem for a nonlinear differential equation in a Hilbert space $H$ :

$$
\dot{z}(t)=-\left[F^{\prime}(z(t))\right]^{-1} F(z(t)) \quad z(0)=z_{0},
$$

where $z_{0}$ is some initial approximation point. In [25] E.P. Zhidkov and I.V. Puzynin applied continuous Newton's method for solving nonlinear physical problems. Y. Alber used continuous methods for solving operator equations and variational inequalities $([6,7,8])$. A modified continuous Newton's method is proposed in $[1,3]$. In this method one avoids numerically difficult inversion of the Fréchet derivative $F^{\prime}$ by solving an expanded system of nonlinear differential equation in Hilbert space. In $[2,3]$ one can find the applications of this method to some physical problems.

The goal of this chapter is to develop a general approach to continuous analogs of discrete methods and to establish fairly general convergence theorems. This approach is based on an analysis of the solution to the Cauchy problem for a nonlinear differential equation in a Hilbert space. Such an analysis was done for well-posed and some ill-posed problems in $[1,4,5]$, and was based on a usage of integral inequalities. 
Let $z_{0}$ be an initial approximation for a solution to (1.1) and $z(t)$ be the trajectory of an autonomous dynamical system:

$$
\dot{z}(t)=\Phi(z(t)), \quad 0 \leq t<\infty, \quad z(0)=z_{0} .
$$

The main question investigated in this chapter is:

Under what assumptions on operators $F$ in (1.1) and $\Phi$ in (1.9) one can guarantee that:

(i) Cauchy problem (1.9) is uniquely solvable for $t \in[0,+\infty)$,

(ii) the solution $z(t)$ tends to one of the solutions of (1.1) as $t \rightarrow \infty$,

(iii) there exists a step $\omega$ (or a sequence $\left\{\omega_{n}\right\}$ ) such that the corresponding discrete (iterative) method $z_{n+1}=z_{n}+\omega \Phi\left(z_{n}\right)$ (or $\left.z_{n+1}=z_{n}+\omega_{n} \Phi\left(z_{n}\right)\right)$ produces the sequence $\left\{z_{n}\right\}$, which converges to one of the solutions of (1.1).

The answers are given in Theorems 2.2, 3.1 and Corollaries 1, 2 .

Thus an analysis of continuous processes is based on the investigation of the asymptotic behavior of nonlinear dynamical systems in Banach and Hilbert spaces. If a convergence theorem is proved for a continuous method, one can construct various discrete schemes generated by this continuous process. Thus construction of a discrete numerical scheme is divided into two parts: construction of the continuous process and numerical integration of the corresponding nonlinear differential equation in a Hilbert space.

The main assumption in Theorems 2.2,3.1 and Corollaries 1, 2 is that the Fréchet derivative $F^{\prime}$ of the operator $F$ has a trivial null-space at the solution to (1.1). If $F^{\prime}$ has a nontrivial null-space at the solution to (1.1) then, in general, one can use the classical Newton method for solving of (1.1) only under some strong special assumptions on the operator $F$ (see $[9,13]$ ). In order to relax these assumptions and to construct numerical method for solving (1.1) when $F^{\prime}$ has a nontrivial nullspace at the solution to (1.1), one needs a regularized discrete Newton-like methods (see $[6,11,15,22,24])$.

In this chapter we consider the continuous Newton's method:

$$
\dot{z}(t)=-\left[F^{\prime}(z(t))+\varepsilon(t) I\right]^{-1}\left[F(z(t))+\varepsilon(t)\left(z(t)-\tilde{z}_{0}\right)\right], \quad z(0)=z_{0},
$$

where $\varepsilon(t)$ is a specially chosen positive function which tends to zero as $t \rightarrow+\infty$.

Thus, in the framework of our general approach, instead of the Cauchy problem (1.9) for autonomous equation the following Cauchy problem for nonautonomous equation has to be considered:

$$
\dot{z}(t)=\Phi(z(t), t), \quad z(0)=z_{0},
$$

where $\Phi$ is a nonlinear operator, $\Phi: H \times[0,+\infty) \rightarrow H$.

An analysis of dynamical system (1.11) is more complicated. In this study we use new integral inequality (Theorem 4.2). Based on Theorem 4.2, a general convergence theorem for a regularized continuous process is proved (Theorem 5.1). Applying this theorem to the regularized Newton's and simple iteration methods (for monotone operators) we obtain convergence theorems under less restrictive conditions on the equation than the theorems known for the corresponding discrete methods. Statements of these theorems contain some useful recommendations for the choice of a regularizing operator and estimate the rate of convergence of the regularized process. Convergence theorems for regularized continuous Newton-like methods are established in $[4,7,22]$. The applications of these scheme to GaussNewton-type methods for nonmonotone operators can be found in $[4,5]$. 
Throughout this chapter the Hilbert space is assumed real-valued.

\section{Continuous methods For Well posed problems}

Let $y$ be a solution to equation (1.1), $z_{0}$ be some point in $H$, considered as an initial approximation to a solution to equation (1.1), and $z(t)$ be some trajectory in $H$ such that $z(0)=z_{0}$.

Definition 2.1. We say that a trajectory $z(t)$ converges to solution $y$ of equation (1.1) exponentially if there exist positive constants $c$ and $c_{1}$ such that

$$
\|z(t)-y\| \leq c_{1} e^{-c t}\left\|F\left(z_{0}\right)\right\|, \quad\|F(z(t))\| \leq\left\|F\left(z_{0}\right)\right\| e^{-c t} .
$$

Consider Cauchy problem (1.9). Examples of the choice of an operator $\Phi$ are given in Remark 1.

Theorem 2.2. Assume that there exist some positive numbers $r, c$ such that $F, F^{\prime}$, and $\Phi$ are Fréchet differentiable and bounded in $B_{r}\left(z_{0}\right)$ and the following conditions hold for every $h \in B_{r}\left(z_{0}\right)$ :

$$
\left(F^{\prime}(h) \Phi(h), F(h)\right) \leq-c\|F(h)\|^{2},
$$

and

$$
\|\Phi(h)\| \leq \frac{r c}{\left\|F\left(z_{0}\right)\right\|}\|F(h)\| .
$$

Then

1) there exists a solution $z=z(t), \quad t \in[0, \infty)$, to problem (1.9) and $z(t) \in$ $B_{r}\left(z_{0}\right)$ for all $t \in[0,+\infty)$;

2) there exists

$$
\lim _{t \rightarrow+\infty} z(t)=y
$$

$y$ is a solution of problem (1.1) in $B_{r}\left(z_{0}\right)$, and $z(t)$ converges to $y$ exponentially.

Remark 2.3. a) Choosing $\Phi(h)=-\left[F^{\prime}(h)\right]^{-1} F(h)$ one gets Continuous Newton's method. In this case $c=1$ and Theorem 2.2 yields the convergence theorem for Continuous Newton's method ([16]);

b) choosing $\Phi=-F$, one gets a simple iteration method for which condition (2.2) means strict monotonicity of $F$;

c) $\Phi(h)=-\left[F^{\prime}(h)\right]^{*} F(h)$ corresponds to the gradient method.

\section{Proof of Theorem 2.2.}

From the Fréchet differentiability of $\Phi$ in $B_{r}\left(z_{0}\right)$, we get the local existence of a solution of the problem (1.8). Then from (1.8) one gets:

$$
F^{\prime}(z(t)) \dot{z}(t)=-F^{\prime}(z(t)) \Phi(z(t) .
$$

From (2.5) denoting $\lambda(t)=F(z(t))$, one gets:

$$
\dot{\lambda}(t)=-F^{\prime}(z(t)) \Phi\left(z(t), \quad \lambda(0)=F\left(z_{0}\right) .\right.
$$

Therefore, for sufficiently small $t$ for which $z(t) \in B_{r}\left(z_{0}\right)$, one can use estimate (2.2) and get:

$$
\frac{d}{d t}\|\lambda(t)\|^{2}=2(\dot{\lambda}(t), \lambda(t))=-2\left(F ^ { \prime } ( z ( t ) ) \Phi \left(z(t), F(z(t)) \leq-2 c\|\lambda(t)\|^{2} .\right.\right.
$$

Thus, the following estimate holds at least for sufficiently small $t>0$ :

$$
\|\lambda(t)\| \leq\left\|F\left(z_{0}\right)\right\| e^{-c t} .
$$


For $0 \leq t_{1} \leq t_{2}$ one has:

$$
\begin{gathered}
\left\|z\left(t_{2}\right)-z\left(t_{1}\right)\right\| \leq\left\|\int_{t_{1}}^{t_{2}} \dot{z}(s) d s\right\| \leq \int_{t_{1}}^{t_{2}}\|\Phi(z(s))\| d s \\
\leq \frac{r c}{\left\|F\left(z_{0}\right)\right\|} \int_{t_{1}}^{t_{2}}\|\lambda(s)\| d s \leq r\left(e^{-c t_{1}}-e^{-c t_{2}}\right) \leq r e^{-c t_{1}} .
\end{gathered}
$$

Setting $t_{1}=0$ and $t_{2}=t$, one concludes from (2.8) that $z(t) \in B_{r}\left(z_{0}\right)$ for all $t>0$. Now let $t_{1}=t$ and $t_{2} \rightarrow+\infty$ in (2.8). Then one gets

$$
\|z(t)-y\| \leq r e^{-c t}
$$

where $y$ is defined in (2.4) and the limit in (2.4) does exist due to (2.8). The exponential convergence of $z(t)$ to $y$ follows now from (2.7) and (2.9).

Remark 2.4. Note that in general the assumptions of Theorem 2.2 do not imply the uniqueness of a solution of equation (1.1) in $B_{r}(y)$. If (1.1) is not uniquely solvable then $z(t)$ converges to one of its solutions.

To establish convergence theorems for discrete methods we need a modified version of the statement of Theorem 2.2. We start with the following definition.

Definition 2.5. Let $y$ be a unique solution to equation (1.1) in some set $M$. $M$ is called $\Phi$-attractive set for $y$ if for any $z_{0} \in M$ the trajectory $z(t)$ of the dynamical system (1.9) does not leave $M$ and tends to $y$ as $t \rightarrow+\infty$. If $z(t)$ converges to $y$ exponentially we call $M$ an exponentially $\Phi$-attractive set for $y$. If constants $c$ and $c_{1}$ in Definition 2.1 do not depend on a point $z_{0} \in M$ we call $M$ a uniformlyexponentially $\Phi$-attractive set for $y$.

Let us formulate a corollary to Theorem 2.2.

Corollary 1. Assume that there exist some positive numbers $r, c$ such that $y$ is the unique solution to problem (1.1) in the ball $B_{r}(y), z_{0} \in B_{r}(y)$, and the assumptions of Theorem2.2 are satisfied in $B_{r}(y)$. Then $B_{r}(y)$ is a uniformlyexponentially $\Phi$-attractive set for $y$ with $c$ defined in (2.2) and

$$
c_{1}=\frac{r}{\left\|F\left(z_{0}\right)\right\|} .
$$

\section{DisCRETIZATION THEOREMS FOR WELL-POSED PROBLEMS}

Consider the following Cauchy problem:

$$
\dot{z}(t)=\Phi(z(t)), \quad z(0)=z_{0},
$$

and the corresponding discrete process:

$$
z_{n+1}=z_{n}+\omega \Phi\left(z_{n}\right) .
$$

Theorem 3.1. Let $B_{r}(y)$ be a uniformly-exponentially $\Phi$-attractive set for $y$ for some $r>0$ such that $r \geq c_{1}\left\|F\left(z_{0}\right)\right\|$, where $c_{1}$ is the same as in Definition 2.1, $z_{0} \in B_{r}(y)$. Assume that

(1) $F$ and $\Phi$ are Fréchet differentiable in $B_{r}(y)$, and

$$
\begin{array}{r}
\|\Phi(h)\| \leq N_{0}\|F(h)\|, \quad\left\|F^{\prime}(h)\right\| \leq N_{1}, \\
\left\|\Phi^{\prime}(h)\right\| \leq N_{2} \quad \text { for } h \in B_{r}(y),
\end{array}
$$


FiguRE 1. Discretization scheme of a continuous process.

(2) $c_{2}$ and $\omega$ are some positive constants satisfying the following inequalities:

$$
\begin{gathered}
\left(c_{1} e^{-c \omega}+N_{0} N_{2} \omega^{2}\right)\left\|F\left(z_{0}\right)\right\| \leq r e^{-c_{2} \omega}, \\
e^{-c \omega}+N_{0} N_{1} N_{2} \omega^{2} \leq e^{-c_{2} \omega} .
\end{gathered}
$$

Then all $\left\{z_{n}\right\}, n=1,2, \ldots$, defined by formula (3.2) belong to the ball $B_{r}(y)$ and

$$
\left\|z_{n}-y\right\| \leq r e^{-c_{2} n \omega}, \quad\left\|F\left(z_{n}\right)\right\| \leq\left\|F\left(z_{0}\right)\right\| e^{-c_{2} n \omega}, \quad n=0,1,2, \ldots
$$

\section{Proof of Theorem 3.1}

The idea of the proof is illustrated in Figure1. Let $z_{0}$ be an initial approximation point. Since $B_{r}(y)$ is an exponentially attractive set for $y$ integral curve $\psi_{0}(t)$ of equation (3.1) connects $z_{0}$ with $y$. Let $\omega$ be a stepsize. Consider points $\psi_{1}(\omega)$ and $z_{1}$ defined by (3.1) and (3.2) correspondingly. The point $\psi_{1}(\omega)$ is located on an integral curve and the point $z_{1}$ is located on a tangent line to this integral curve passing through $z_{0}$. Since $\psi_{1}(t)$ converges exponentially to $y$ and the distance between $\psi_{1}(\omega)$ and $z_{1}$ decreases as $\omega^{2}$ for $\omega \rightarrow 0$, one shows that for a sufficiently small step $\omega, z_{1}$ is closer to $y$ than $z_{0}$. Therefore $z_{1}$ also belongs to $B_{r}(y)$. Moreover using the triangle inequality one can estimate the distance between $z_{1}$ and $y$. Then take the integral curve $\psi_{2}(t)$, which connects $z_{1}$ with $y$, points $\psi_{2}(\omega)$ and $z_{2}$, show that $z_{2}$ belongs to $B_{r}(y)$, and estimate the distance between $z_{2}$ and $y$. Repeating this process one estimates the distance between $\left\{z_{n}\right\}$ and $y$ and shows that this distance exponentially tends to zero.

We prove (3.6) using mathematical induction. For $n=0$ conditions (3.6) are satisfied. Assume that (3.6) are satisfied for $n=m-1$.

Denote by $\psi_{m}(t)$ the solution to the following Cauchy problem:

$$
\dot{z}(t)=\Phi(z(t)), \quad 0<t \leq \omega, \quad z(0)=z_{m-1} .
$$

Then one has:

$$
\left\|z_{m}-y\right\| \leq\left\|\psi_{m}(\omega)-y\right\|+\left\|\psi_{m}(\omega)-z_{m}\right\| .
$$

Since $B_{r}(y)$ is a uniform-exponentially $\Phi$-attractive set for $y$ one gets:

$$
\left\|F\left(\psi_{m}(t)\right)\right\| \leq\left\|F\left(z_{m-1}\right)\right\| e^{-c t}, \quad t \in[0, \omega],
$$

and

$$
\left\|\psi_{m}(\omega)-y\right\| \leq c_{1} e^{-c \omega}|| F\left(z_{m-1}\right)\left\|\leq c_{1} e^{-c \omega} e^{-c_{2} \omega(m-1)}\right\| F\left(z_{0}\right) \| .
$$

From (3.1) and (3.2) one obtains:

$$
\begin{gathered}
\psi_{m}(\omega)-z_{m}=\int_{0}^{\omega}\left[\Phi(z(\tau))-\Phi\left(z_{m-1}\right)\right] d \tau=\int_{0}^{\omega} d \tau \int_{0}^{1} \frac{d}{d s} \Phi(z(s \tau)) d s= \\
=\int_{0}^{\omega} \tau d \tau \int_{0}^{1} \Phi^{\prime}(z(s \tau)) \dot{z}(s \tau) d s
\end{gathered}
$$


Therefore

$$
\left\|\psi_{m}(\omega)-z_{m}\right\| \leq \omega \int_{0}^{\omega}\left\|\Phi^{\prime}(z(s))\right\|\|\Phi(z(s))\| d s .
$$

Using (3.9) one gets:

$$
\begin{gathered}
\left\|\psi_{m}(\omega)-z_{m}\right\| \leq \omega N_{0} N_{2} \int_{0}^{\omega}\|F(z(s))\| d s \\
\leq \omega^{2} N_{0} N_{2}\left\|F\left(z_{m-1}\right)\right\| \leq \omega^{2} N_{0} N_{2} e^{-c_{2} \omega(m-1)}\left\|F\left(z_{0}\right)\right\| .
\end{gathered}
$$

From (3.8), (3.10), and (3.13) one gets:

$$
\left\|z_{m}-y\right\| \leq\left(c_{1} e^{-c \omega} e^{-c_{2} \omega(m-1)}+\omega^{2} N_{0} N_{2} e^{-c_{2} \omega(m-1)}\right)\left\|F\left(z_{0}\right)\right\| .
$$

Using condition (2.10) one obtains:

$$
\left\|z_{m}-y\right\| \leq r e^{-c_{2} m \omega}
$$

Also

$$
\begin{aligned}
& \left\|F\left(z_{m}\right)\right\| \leq\left\|F\left(\psi_{m}(\omega)\right)\right\|+N_{1}\left\|\psi_{m}(\omega)-z_{m}\right\| \leq e^{-c \omega}\left\|F\left(z_{m-1}\right)\right\| \\
& +\omega^{2} N_{0} N_{1} N_{2} e^{-c_{2} \omega(m-1)}\left\|F\left(z_{0}\right)\right\| \leq e^{-c_{2} \omega(m-1)}\left(e^{-c \omega}+\omega^{2} N_{0} N_{1} N_{2}\right)\left\|F\left(z_{0}\right)\right\| \\
& \leq\left(e^{-c \omega}+\omega^{2} N_{0} N_{1} N_{2}\right) e^{-c_{2} \omega(m-1)}\left\|F\left(z_{0}\right)\right\| .
\end{aligned}
$$

From (3.16) and (3.5) one gets:

$$
\left\|F\left(z_{m}\right)\right\| \leq\left\|F\left(z_{0}\right)\right\| e^{-c_{2} m \omega} .
$$

Thus the estimate (2.10) and (3.5) hold also for $n=m$. Theorem 3.1 is proved.

Corollary 2. Assume that there exist some positive numbers $r, c$ such that:

(1) $y$ is the unique solution to problem (1.1) in the ball $B_{r}(y)$, and an initial approximation point $z_{0} \in B_{r}(y)$,

(2) the assumptions of Theorem2.2 are satisfied in $B_{r}(y)$,

$$
\left\|F^{\prime}(h)\right\| \leq N_{1},\left\|\Phi^{\prime}(h)\right\| \leq N_{2} \quad \text { for } \quad h \in B_{r}(y),
$$

(4) $c_{2}$ and $\omega$ are some positive constants satisfying the following inequality:

$$
e^{-c \omega}+c N_{2} \omega^{2} \max \left\{1, \frac{r N_{1}}{\left\|F\left(z_{0}\right)\right\|}\right\} \leq e^{-c_{2} \omega},
$$

(5) $z_{0}$ is an initial approximation point and the sequence $\left\{z_{n}\right\}, n=1,2, \ldots$, is defined recursively:

$$
z_{n}=z_{n-1}+\omega \Phi\left(z_{n-1}\right),
$$

Then all $\left\{z_{n}\right\}, n=1,2, \ldots$, belong to the ball $B_{r}(y)$ and

$$
\left\|z_{n}-y\right\| \leq r e^{-c_{2} n \omega}, \quad\left\|F\left(z_{n}\right)\right\| \leq\left\|F\left(z_{0}\right)\right\| e^{-c_{2} n \omega}, \quad n=0,1,2, \ldots
$$

\section{Proof of Corollary 2.}

Since the assumptions of Theorem 2.2 are satisfied in $B_{r}(y)$, choosing

$$
N_{0}=\frac{r c}{\left\|F\left(z_{0}\right)\right\|}
$$


one gets the first inequality in (3.3) from (2.3). The second and the third inequalities in (3.3) follow from (3.18).

From Corollary 1 one gets that $B_{r}(y)$ is a uniformly-exponentially $\Phi$-attractive set for $y$ with $c$ defined in condition $(2.2)$ and

$$
c_{1}=\frac{r}{\left\|F\left(z_{0}\right)\right\|} .
$$

For such $N_{0}$ and $c_{1}$ condition (3.19) is equivalent to conditions (2.10) and (3.5). To finish the proof one refers to Theorem 3.1.

\section{NONLINEAR INTEGRAL INEQUALITY}

The main result of this section is Theorem 4.2 which is used throughout the paper.

The following lemma is a version of some known results concerning integral inequalities (see e.g. Theorem 22.1 in [23]). For convenience of the reader and to make the presentation essentially self-contained we include a proof.

Lemma 4.1. Let $f(t, w), g(t, u)$ be continuous on region $[0, T) \times D(D \subset R$, $T \leq \infty)$ and $f(t, w) \leq g(t, u)$ if $w \leq u, t \in(0, T), w, u \in D$. Assume that $g(t, u)$ is such that the Cauchy problem

$$
\dot{u}=g(t, u), \quad u(0)=u_{0}, \quad u_{0} \in D
$$

has a unique solution. If

$$
\dot{w} \leq f(t, w), \quad w(0)=w_{0} \leq u_{0}, \quad w_{0} \in D,
$$

then $u(t) \geq w(t)$ for all $t$ for which $u(t)$ and $w(t)$ are defined.

\section{Proof of Lemma 4.1}

Step 1. Suppose first $f(t, w)<g(t, u)$, if $w \leq u$. Since $w_{0} \leq u_{0}$ and $\dot{w}(0) \leq$ $f\left(\overline{\left.t, w_{0}\right)<g}\left(t, u_{0}\right)=\dot{u}(0)\right.$, there exists $\delta>0$ such that $u(t)>w(t)$ on $(0, \delta]$. Assume that for some $t_{1}>\delta$ one has $u\left(t_{1}\right)<w\left(t_{1}\right)$. Then for some $t_{2}<t_{1}$ one has

$$
u\left(t_{2}\right)=w\left(t_{2}\right) \text { and } u(t)<w(t) \text { for } t \in\left(t_{2}, t_{1}\right] .
$$

One gets

$$
\dot{w}\left(t_{2}\right) \geq \dot{u}\left(t_{2}\right)=g\left(t, u\left(t_{2}\right)\right)>f\left(t, w\left(t_{2}\right)\right) \geq \dot{w}\left(t_{2}\right) .
$$

This contradiction proves that there is no point $t_{2}$ such that $u\left(t_{2}\right)=w\left(t_{2}\right)$.

Step 2. Now consider the case $f(t, w) \leq g(t, u)$, if $w \leq u$. Define

$$
\dot{u}_{n}=g\left(t, u_{n}\right)+\varepsilon_{n}, \quad u_{n}(0)=u_{0}, \quad \varepsilon_{n}>0, \quad n=0,1, \ldots,
$$

where $\varepsilon_{n}$ tends monotonically to zero. Then

$$
\dot{w} \leq f(t, w) \leq g(t, u)<g(t, u)+\varepsilon_{n}, \quad w \leq u .
$$

By Step $1 u_{n}(t) \geq w(t), n=0,1, \ldots$. Fix an arbitrary compact set $\left[0, T_{1}\right], 0<T_{1}<$ $T$.

$$
u_{n}(t)=u_{0}+\int_{0}^{t} g\left(\tau, u_{n}(\tau)\right) d \tau+\varepsilon_{n} t .
$$

Since $g(t, u)$ is continuous, the sequence $\left\{u_{n}\right\}$ is uniformly bounded and equicontinuous on $\left[0, T_{1}\right]$. Therefore there exists a subsequence $\left\{u_{n_{k}}\right\}$ which converges 
uniformly to a continuous function $u(t)$. By continuity of $g(t, u)$ we can pass to the limit in (4.3) and get

$$
u(t)=u_{0}+\int_{0}^{t} g(\tau, u(\tau)) d \tau, \quad t \in\left[0, T_{1}\right] .
$$

Since $T_{1}$ is arbitrary (4.4) is equivalent to the initial Cauchy problem that has a unique solution. The inequality $u_{n_{k}}(t) \geq w(t), k=0,1, \ldots$ implies $u(t) \geq w(t)$. If the solution to the Cauchy problem (4.1) is not unique, the inequality $w(t) \leq u(t)$ holds for the maximal solution to (4.1).

The following theorem is a key to the basic result, namely to Theorem 5.1.

Theorem 4.2. Let $\gamma(t), \sigma(t), \beta(t) \in C\left[t_{0}, \infty\right)$ for some real number $t_{0}$. If there exists a positive function $\mu(t) \in C^{1}\left[t_{0}, \infty\right)$ such that

$$
0 \leq \sigma(t) \leq \frac{\mu(t)}{2}\left(\gamma(t)-\frac{\dot{\mu}(t)}{\mu(t)}\right), \quad \beta(t) \leq \frac{1}{2 \mu(t)}\left(\gamma(t)-\frac{\dot{\mu}(t)}{\mu(t)}\right),
$$

then a nonnegative solution to the following inequalities:

$$
\dot{v}(t) \leq-\gamma(t) v(t)+\sigma(t) v^{2}(t)+\beta(t), \quad v\left(t_{0}\right)<\frac{1}{\mu\left(t_{0}\right)},
$$

satisfies the estimate:

$$
v(t) \leq \frac{1-\nu(t)}{\mu(t)}<\frac{1}{\mu(t)}
$$

for all $t \in\left[t_{0}, \infty\right)$, where

$$
\nu(t)=\left(\frac{1}{1-\mu\left(t_{0}\right) v\left(t_{0}\right)}+\frac{1}{2} \int_{t_{0}}^{t}\left(\gamma(s)-\frac{\dot{\mu}(s)}{\mu(s)}\right) d s\right)^{-1} .
$$

Remark 4.3. Without loss of generality one can assume $\beta(t) \geq 0$.

In [8] a differential inequality $\dot{v} \leq-A(t) \psi(v(t))+\beta(t)$ was studied under some assumptions which include, among others, the positivity of $\psi(v)$ for $v>0$. In Theorem 4.2 the term $-\gamma(t) v(t)+\sigma(t) v^{2}(t)$ (which is analogous to some extent to the term $-A(t) \psi(v(t)))$ can change sign. Our Theorem 4.2 is not covered by the result in [8]. In particular, in Theorem 4.2 an analog of $\psi(v)$, for the case $\gamma(t)=\sigma(t)=A(t)$, is the function $\psi(v):=v-v^{2}$. This function goes to $-\infty$ as $v$ goes to $+\infty$, so it does not satisfy the positivity condition imposed in [8].

Unlike in the case of Bihari integral inequality ([10]) one cannot separate variables in the right hand side of the first inequality (4.6) and estimate $v(t)$ by a solution of the Cauchy problem for a differential equation with separating variables. The proof below is based on a special choice of the solution to the Riccati equation majorizing a solution of inequality (4.6).

\section{Proof of Theorem 4.2.}

Denote:

$$
w(t):=v(t) e^{\int_{t_{0}}^{t} \gamma(s) d s}
$$

then (4.6) implies:

$$
\dot{w}(t) \leq a(t) w^{2}(t)+b(t), \quad w\left(t_{0}\right)=v\left(t_{0}\right),
$$


where

$$
a(t)=\sigma(t) e^{-\int_{t_{0}}^{t} \gamma(s) d s}, \quad b(t)=\beta(t) e^{\int_{t_{0}}^{t} \gamma(s) d s} .
$$

Consider Riccati's equation:

$$
\dot{u}(t)=\frac{\dot{f}(t)}{g(t)} u^{2}(t)-\frac{\dot{g}(t)}{f(t)} .
$$

One can check by a direct calculation that the the solution to problem (4.11) is given by the following formula [17, eq. 1.33]:

$$
u(t)=-\frac{g(t)}{f(t)}+\left[f^{2}(t)\left(C-\int_{t_{0}}^{t} \frac{\dot{f}(s)}{g(s) f^{2}(s)} d s\right)\right]^{-1} .
$$

Define $f$ and $g$ as follows:

$$
f(t):=\mu^{\frac{1}{2}}(t) e^{-\frac{1}{2} \int_{t_{0}}^{t} \gamma(s) d s}, \quad g(t):=-\mu^{-\frac{1}{2}}(t) e^{\frac{1}{2} \int_{t_{0}}^{t} \gamma(s) d s},
$$

and consider the Cauchy problem for equation (4.11) with the initial condition $u\left(t_{0}\right)=v\left(t_{0}\right)$. Then $C$ in (4.12) takes the form:

$$
C=\frac{1}{\mu\left(t_{0}\right) v\left(t_{0}\right)-1}
$$

From (4.5) one gets

$$
a(t) \leq \frac{\dot{f}(t)}{g(t)}, \quad b(t) \leq-\frac{\dot{g}(t)}{f(t)}
$$

Since $f g=-1$ one has:

$$
\int_{t_{0}}^{t} \frac{\dot{f}(s)}{g(s) f^{2}(s)} d s=-\int_{t_{0}}^{t} \frac{\dot{f}(s)}{f(s)} d s=\frac{1}{2} \int_{t_{0}}^{t}\left(\gamma(s)-\frac{\dot{\mu}(s)}{\mu(s)}\right) d s .
$$

Thus

$$
u(t)=\frac{e^{\int_{t_{0}}^{t} \gamma(s) d s}}{\mu(t)}\left[1-\left(\frac{1}{1-\mu\left(t_{0}\right) v\left(t_{0}\right)}+\frac{1}{2} \int_{t_{0}}^{t}\left(\gamma(s)-\frac{\dot{\mu}(s)}{\mu(s)}\right) d s\right)^{-1}\right]
$$

It follows from conditions (4.5) and from the second inequality in (4.6) that the solution to problem (4.11) exists for all $t \in[0, \infty)$ and the following inequality holds with $\nu(t)$ defined by (4.8):

$$
1>1-\nu(t) \geq \mu\left(t_{0}\right) v\left(t_{0}\right) .
$$

From Lemma 4.1 and from formula (4.14) one gets:

$$
v(t) e^{\int_{t_{0}}^{t} \gamma(s) d s}:=w(t) \leq u(t)=\frac{1-\nu(t)}{\mu(t)} e^{\int_{t_{0}}^{t} \gamma(s) d s}<\frac{1}{\mu(t)} e^{\int_{t_{0}}^{t} \gamma(s) d s},
$$

and thus estimate (4.7) is proved.

To illustrate conditions of Theorem 4.2 consider the following examples of functions $\gamma, \sigma, \beta$, satisfying (4.5) for $t_{0}=0$.

Example 4.4. Let

$$
\gamma(t)=c_{1}(1+t)^{\nu_{1}}, \quad \sigma(t)=c_{2}(1+t)^{\nu_{2}}, \quad \beta(t)=c_{3}(1+t)^{\nu_{3}}
$$

where $c_{2}>0, c_{3}>0$. Choose $\mu(t):=c(1+t)^{\nu}, c>0$. From (4.5), (4.6) one gets the following conditions

$$
c_{2} \leq \frac{c c_{1}}{2}(1+t)^{\nu+\nu_{1}-\nu_{2}}-\frac{c \nu}{2}(1+t)^{\nu-1-\nu_{2}},
$$


DYNAMICAL SYSTEMS AND DISCRETE METHODS FOR SOLVING NONLINEAR ILL-POSED PROBLEM\$

$$
c_{3} \leq \frac{c_{1}}{2 c}(1+t)^{\nu_{1}-\nu-\nu_{3}}-\frac{\nu}{2 c}(1+t)^{-\nu-1-\nu_{3}}, \quad c v(0)<1 .
$$

Thus one obtains the following conditions:

$$
\nu_{1} \geq-1, \quad \nu_{2}-\nu_{1} \leq \nu \leq \nu_{1}-\nu_{3}
$$

and

$$
c_{1}>\nu, \quad \frac{2 c_{2}}{c_{1}-\nu} \leq c \leq \frac{c_{1}-\nu}{2 c_{3}}, \quad c v(0)<1 .
$$

Therefore for such $\gamma, \sigma, \beta$ a function $\mu$ with the desired properties exists if

$$
\nu_{1} \geq-1, \quad \nu_{2}+\nu_{3} \leq 2 \nu_{1},
$$

and

$$
c_{1}>\nu_{2}-\nu_{1}, \quad 2 \sqrt{c_{2} c_{3}} \leq c_{1}+\nu_{1}-\nu_{2}, \quad 2 c_{2} v(0)<c_{1}+\nu_{1}-\nu_{2} .
$$

In this case one can choose $\nu=\nu_{2}-\nu_{1}, c=\frac{2 c_{2}}{c_{1}+\nu_{1}-\nu_{2}}$. However in order to have $v(t) \rightarrow 0$ as $t \rightarrow+\infty$ (the case of interest in Theorem 5.1) one needs the following conditions:

$$
\nu_{1} \geq-1, \quad \nu_{2}+\nu_{3} \leq 2 \nu_{1}, \quad \nu_{1}>\nu_{3}
$$

and

$$
c_{1}>\nu_{2}-\nu_{1}, \quad 2 \sqrt{c_{2} c_{3}} \leq c_{1}, \quad 2 c_{2} v(0)<c_{1} .
$$

Example 4.5. If

$$
\gamma(t)=\gamma_{0}, \quad \sigma(t)=\sigma_{0} e^{\nu t}, \quad \beta(t)=\beta_{0} e^{-\nu t}, \quad \mu(t)=\mu_{0} e^{\nu t},
$$

then conditions (4.5), (4.6) are satisfied if

$$
0 \leq \sigma_{0} \leq \frac{\mu_{0}}{2}\left(\gamma_{0}-\nu\right), \quad \beta_{0} \leq \frac{1}{2 \mu_{0}}\left(\gamma_{0}-\nu\right), \quad \mu_{0} v(0)<1 .
$$

Example 4.6. Here and throughout the paper log stands for the natural logarithm. For some $t_{1}>0$

$$
\gamma(t)=\frac{1}{\sqrt{\log \left(t+t_{1}\right)}}, \quad \mu(t)=c \log \left(t+t_{1}\right),
$$

conditions (4.5), (4.6) are satisfied if

$$
\begin{gathered}
0 \leq \sigma(t) \leq \frac{c}{2}\left(\sqrt{\log \left(t+t_{1}\right)}-\frac{1}{t+t_{1}}\right), \\
\beta(t) \leq \frac{1}{2 c \log ^{2}\left(t+t_{1}\right)}\left(\sqrt{\log \left(t+t_{1}\right)}-\frac{1}{t+t_{1}}\right), \quad v(0) c \log t_{1}<1 .
\end{gathered}
$$

In all considered examples $\mu(t)$ can tend to infinity as $t \rightarrow+\infty$ and provide a decay of a nonnegative solution to integral inequality (4.6) even if $\sigma(t)$ tends to infinity. Moreover in the first and the third examples $v(t)$ tends to zero as $t \rightarrow+\infty$ when $\gamma(t) \rightarrow 0$ and $\sigma(t) \rightarrow+\infty$. 


\section{Regularization PROCEDURE FOR ILL-POSED PROBLEMS}

In the well-posed case (when the Fréchet derivative $F^{\prime}$ of the operator $F$ is a bijection in a neighborhood of the solution of equation (1.1)) in order to solve equation (1.1) one can use the following continuous processes:

- simple iteration method:

$$
\dot{z}(t)=-F(z(t)), \quad z(0)=z_{0} \in H,
$$

- Newton's method:

$$
\dot{z}(t)=-\left[F^{\prime}(z(t))\right]^{-1} F(z(t)), \quad z(0)=z_{0} \in H .
$$

However if $F^{\prime}$ is not continuously invertible (ill-posed case) one has to replace the Cauchy problems (5.1) and (5.2) by the corresponding regularized Cauchy problems (see $[4,5,6])$ :

- regularized simple iteration method:

$$
\dot{z}(t)=-\left[F(z(t))+\varepsilon(t)\left(z(t)-\tilde{z}_{0}\right)\right], \quad z(0)=z_{0} \in H,
$$

- regularized Newton's method:

$$
\dot{z}(t)=-\left[F^{\prime}(z(t))+\varepsilon(t) I\right]^{-1}\left[F(z(t))+\varepsilon(t)\left(z(t)-\tilde{z}_{0}\right)\right], \quad z(0)=z_{0} \in H .
$$

Here $\tilde{z}_{0} \in H$ is some element, $\varepsilon(t)>0$ is a suitable function, with the properties specified in Theorems 7.12 and 7.22 , and $I$ is the identity operator. The equations in (5.3) and (5.4) are no longer autonomous.

Our goal is to develop a uniform approach to such regularized methods. Let us consider the Cauchy problem:

$$
\dot{z}(t)=\Phi(z(t), t), \quad z(0)=z_{0} \in H,
$$

with an operator $\Phi: H \times[0, \infty) \rightarrow H$.

Let $y$ be a solution to equation (1.1) (as it was mentioned in Introduction, we assume that this equation is solvable). Denote: $B_{R}(y):=\{h: h \in H,\|h-y\|<R\}$.

Theorem 5.1. Assume that there exists $r>0$ such that $\Phi(h, t)$ is Fréchet differentiable with respect to $h$ in the ball $B_{2 r}(y)$ for any $t \in[0, \infty)$ and satisfy the following condition:

there exists a differentiable function $x(t), x:[0,+\infty) \rightarrow B_{r}(y)$, such that for any $h \in B_{2 r}(y), t \in[0,+\infty)$

$$
(\Phi(h, t), h-x(t)) \leq \alpha(t)\|h-x(t)\|-\gamma(t)\|h-x(t)\|^{2}+\sigma(t)\|h-x(t)\|^{3},
$$

where $\alpha(t)$ is a continuous function, $\alpha(t) \geq 0, \gamma(t)$ and $\sigma(t)$ satisfy conditions (4.5) of Theorem 4.2 with

$$
\beta(t):=\|\dot{x}(t)\|+\alpha(t)
$$

$\mu(t)$ in (4.5) tends to $+\infty$ as $t \rightarrow+\infty$, and

$$
\left\|z_{0}-x(0)\right\|<\frac{1}{\mu(0)}, \quad \inf _{t \in[0,+\infty)} \mu(t) \geq \frac{1}{r} .
$$

Then problem (5.5) has a unique solution $z(t) \in B_{2 r}(y)$ for all $t \in[0, \infty)$, and

$$
\|z(t)-x(t)\|<\frac{1-\nu(t)}{\mu(t)}<\frac{1}{\mu(t)},
$$


where $\nu(t)$ is defined by (4.8), and

$$
\lim _{t \rightarrow+\infty}\|z(t)-x(t)\|=0 .
$$

Remark 5.2. One can choose the regularizing operator $\Phi(h, t)$ in (5.5) such that condition (5.6) holds in the case when $F^{* *}(h) F^{\prime}(h)$ is not boundedly invertible (see Sect. 7, Lemmas 7.11, 7.20).

\section{Proof of Theorem 5.1}

Since $\Phi(h, t)$ is Fréchet differentiable with respect to $h$ problem (5.5) is locally uniquely solvable. Denote by $[0, T)$ the maximal interval on which the solution $z(t)$ to problem (5.5) exists and $z(t) \in B_{2 r}(y)$. One has to show that $T=+\infty$. Assume $T<+\infty$, then the trajectory $z(t)$ hits the boundary of $B_{2 r}(y)$ at $t=T$ : $\|z(T)-y\|=2 r$. Since $H$ is a real Hilbert space one has:

$\frac{1}{2} \frac{d}{d t}\|z(t)-x(t)\|^{2}=(\dot{z}-\dot{x}, z(t)-x(t))=(\Phi(z(t), t), z(t)-x(t))-(\dot{x}, z(t)-x(t))$.

Therefore from (5.6) and (5.7) for $t \in[0, T)$ one obtains

$$
\frac{1}{2} \frac{d}{d t}\|z(t)-x(t)\|^{2} \leq-\gamma\|z(t)-x(t)\|^{2}+\sigma(t)\|z(t)-x(t)\|^{3}+\beta(t)\|z(t)-x(t)\| .
$$

Denote

From (5.12) one has:

$$
v(t):=\|z(t)-x(t)\| .
$$

$$
v(t) \dot{v}(t) \leq-\gamma(t) v^{2}(t)+\sigma(t) v^{3}(t)+\beta(t) v(t) .
$$

If $v>0$, one gets:

$$
\dot{v}(t) \leq-\gamma(t) v(t)+\sigma(t) v^{2}(t)+\beta(t) .
$$

If $v=0$ on some interval, then inequality (5.13) is satisfied trivially because $\beta(t) \geq$ 0 . Thus (5.13) holds for all $t>0$.

By Theorem 4.2 using (5.8) one obtains

$$
\|z(t)-x(t)\| \leq \frac{1}{\mu(t)} \leq r, \quad \text { for } \quad t \in[0, T) .
$$

Thus, since $x(t) \in B_{r}(y)$, one gets

$$
\|z(t)-y\| \leq\|z(t)-x(t)\|+\|x(t)-y\|<2 r, \quad \text { for } \quad t \in[0, T) .
$$

Therefore there exists a sequence $\left\{t_{n}\right\} \rightarrow T$ such that $\left\{z\left(t_{n}\right)\right\}$ converges weakly to some $z^{*}$. From equation (5.5) one derives the uniform boundedness of the norm $\|\dot{z}(t)\|$ on $[0, T)$ since $\|\Phi(z(t), t)\| \leq$ const $<\infty$ for $\|z(t)\| \leq$ const and $0 \leq t \leq$ const. Thus there exists $\lim _{t \rightarrow T}\left\|z(t)-z^{*}\right\|=0$. Since

$$
\left\|z^{*}-y\right\| \leq\left\|z^{*}-x(T)\right\|+\|x(T)-y\|<2 r \quad \text { for } \quad t \in[0, T),
$$

the conditions for the unique local solvability of the Cauchy problem for equation (5.5) with initial condition $z(T)=z^{*}$ are satisfied. Therefore one can continue the solution to (5.5) through the point $T$. This contradicts the assumption of maximality of $T$, thus $T=+\infty$. Moreover, from (4.7) one gets:

$$
\lim _{t \rightarrow+\infty}\|z(t)-x(t)\| \leq \lim _{t \rightarrow+\infty} \frac{1}{\mu(t)}=0 .
$$


In order to establish the discretization theorem in the next section we need to estimate $\|\Phi\|$ along the trajectory $z(t)$. The following theorem gives such an estimate.

Theorem 5.3. Let the assumptions of Theorem 5.1 hold and $z(t)$ solve problem (5.5). Assume that the following two conditions hold:

(1) for any $h$ belonging to the trajectory $z(t), \Phi(h, t)$ is differentiable with respect to $t$ and the following two inequalities hold:

$$
\begin{gathered}
\left(\Phi^{\prime}(h, t) \xi, \xi\right) \leq\left(-a_{1}(t)+a_{2}(t)\|\Phi(h, t)\|\right)\|\xi\|^{2} \text { for any } \xi \in H, \\
\left\|\frac{\partial \Phi}{\partial t}(h, t)\right\| \leq \beta_{1}(t)+b_{1}(t)\|\Phi(h, t)\|+b_{2}(t)\|\Phi(h, t)\|^{2},
\end{gathered}
$$

where $\Phi^{\prime}(h, t)$ is the Fréchet derivative with respect to $h, \partial \Phi / \partial t$ is the derivative with respect to $t, a_{1}(t), a_{2}(t), \beta_{1}(t), b_{1}(t)$, and $b_{2}(t)$ are continuous functions;

(2) the functions $\gamma_{1}(t):=a_{1}(t)-b_{1}(t), \sigma_{1}(t):=a_{2}(t)+b_{2}(t)$ and $\beta_{1}(t)$ are continuous and satisfy conditions (4.5) of Theorem 4.2 with $v_{1}(0):=\left\|\Phi\left(z_{0}, 0\right)\right\|<$ $\frac{1}{\mu_{1}(0)}$ and with $\mu_{1}(t)>0$, which tends to $+\infty$ as $t \rightarrow+\infty$.

Then the following estimate holds:

$$
\|\Phi(z(t), t)\| \leq \frac{1}{\mu_{1}(t)} .
$$

\section{Proof of Theorem 5.3.}

Denote $v_{1}(t):=\|\Phi(z(t), t)\|$. Recall that $H$ is a real Hilbert space. From (5.5) one gets:

$$
v_{1} \frac{d v_{1}}{d t}=\left(\frac{d}{d t} \Phi(z(t), t), \Phi(z(t), t)\right)=\left(\frac{\partial \Phi}{\partial t}+\Phi^{\prime}(z(t), t) \dot{z}(t), \Phi(z(t), t)\right) .
$$

From (5.21) and (5.5) one gets:

$$
v_{1} \frac{d v_{1}}{d t}=\left(\Phi^{\prime}(z(t), t) \Phi(z(t), t), \Phi(z(t), t)\right)+\left(\frac{\partial \Phi}{\partial t}, \Phi(z(t), t)\right) .
$$

Using (5.18) and (5.19) one obtains from (5.22) the following inequality:

$$
v_{1} \dot{v}_{1} \leq\left(-a_{1}(t)+a_{2}(t) v_{1}\right) v_{1}^{2}+\left(\beta_{1}(t)+b_{1}(t) v_{1}+b_{2}(t) v_{1}^{2}\right) v_{1}
$$

or

$$
\dot{v}_{1} \leq \beta_{1}+\left(b_{1}-a_{1}\right) v_{1}+\left(a_{2}+b_{2}\right) v_{1}^{2}=\beta_{1}-\gamma_{1} v_{1}+\sigma_{1} v_{1}^{2}
$$

where $\gamma_{1}(t)=a_{1}(t)-b_{1}(t)$ and $\sigma_{1}(t)=a_{2}(t)+b_{2}(t)$.

It follows from the assumptions of Theorem 5.3 that $v_{1}(t)$ satisfies the integral inequality:

$$
\frac{d v_{1}}{d t} \leq-\gamma_{1}(t) v_{1}(t)+\sigma_{1}(t) v_{1}^{2}(t)+\beta_{1}(t)
$$

To finish the proof of Theorem 5.3 one uses Theorem 4.2. 


\section{Discretization theorem For ILl-Posed PRoblems}

The theorem of this section gives an answer to the following question: under what assumptions on $\Phi$ and $\left\{\omega_{n}\right\}$ the convergence of a continuous process

$$
\dot{z}(t)=\Phi(z(t), t), \quad z(0)=z_{0},
$$

implies the convergence of the corresponding discrete process

$$
\begin{gathered}
z_{n}=z_{n-1}+\omega_{n} \Phi\left(z_{n-1}, t_{n-1}\right), \\
t_{n}=t_{n-1}+\omega_{n}, \quad t_{0}=0, \quad n=1,2, \ldots,
\end{gathered}
$$

where $z_{0}$ in (94) is the same as in (6.1).

Theorem 6.1. Let $\Phi$ satisfy conditions of Theorems 5.1 and 5.3 with a function $x(t)$, which tends to $y$ as $t \rightarrow+\infty$.

Assume that

(1)

$$
\left\|\Phi^{\prime}(h, t)\right\| \leq a_{3}(t)+a_{2}(t)\|\Phi(h, t)\|,
$$

where $a_{3}(t)$ is a nonnegative continuous function and $a_{2}(t)$ is the same as in Theorem 5.3;

(2) the sequence $\left\{z_{n}\right\}$ is defined by formulas (6.2) and (6.3);

$$
A=\sup _{t \in[0,+\infty)}\left(a_{1}(t)+a_{3}(t)\right)<+\infty,
$$

where $a_{1}(t)$ is defined in (5.18);

$$
0<\omega_{n} \leq \frac{1}{A}, \quad \sum_{n=1}^{\infty} \omega_{n}=\infty
$$

$$
\frac{\omega_{n}}{\nu_{n}\left(\omega_{n}\right)}<\frac{\mu_{1}\left(t_{n}\right)}{\mu\left(t_{n}\right)},
$$

where

$$
\frac{1}{\nu_{n}(t)}=\frac{1}{1-\mu\left(t_{n-1}\right)\left\|z_{n-1}-x\left(t_{n-1}\right)\right\|}+\frac{1}{2} \int_{t_{n-1}}^{t}\left(\gamma(s)-\frac{\dot{\mu}(s)}{\mu(s)}\right) d s,
$$

the continuous positive functions $\mu(t), \mu_{1}(t)$ are defined in Theorems 5.1 and 5.3;

(6) the function $\mu_{1}(t)$ is monotonically increasing.

Then the following conclusions hold:

i) all $z_{n}, n=1,2, \ldots$, belong to the ball $B_{2 r}(y)$;

ii)

$$
\left\|z_{n}-x\left(t_{n}\right)\right\|<\frac{1}{\mu\left(t_{n}\right)}
$$

iii)

$$
t_{n} \rightarrow+\infty, \quad \frac{1}{\mu\left(t_{n}\right)} \rightarrow 0 \quad \text { as } \quad n \rightarrow \infty
$$


iv)

$$
\lim _{n \rightarrow \infty}\left\|z_{n}-y\right\|=0
$$

\section{Proof of Theorem 6.1.}

Statements (6.10) follow from (6.6) and from the assumption that $\mu_{1}(t) \rightarrow \infty$ as $t \rightarrow \infty$. We prove (6.9) by induction. It follows from (5.8) that $\left\|z_{0}-x(0)\right\|<\frac{1}{\mu(0)}$. Suppose that

$$
\left\|z_{n-1}-x\left(t_{n-1}\right)\right\|<\frac{1}{\mu\left(t_{n-1}\right)} .
$$

We want to prove that (6.12) with $n$ replacing $n-1$ is true. Denote by $\psi_{n}(t)$ the solution to the following Cauchy problem:

$$
\dot{z}(t)=\Phi(z(t), t), \quad t_{n-1}<t \leq t_{n}, \quad z\left(t_{n-1}\right)=z_{n-1} .
$$

For problem (6.13) the conditions of Theorem 5.1 are satisfied by assumption. Therefore from (5.9) one gets:

$$
\left\|\psi_{n}(t)-x(t)\right\| \leq \frac{1-\nu_{n}(t)}{\mu(t)}
$$

with $\nu_{n}(t)$ defined in (6.8). Using (6.1) and (6.2) one gets:

$$
\begin{gathered}
\psi_{n}\left(t_{n}\right)-z_{n}=\int_{t_{n-1}}^{t_{n}}\left[\Phi(z(\tau), \tau)-\Phi\left(z_{n-1}, t_{n-1}\right)\right] d \tau= \\
=\int_{t_{n-1}}^{t_{n}} d \tau \int_{0}^{1} \frac{d}{d s} \Phi\left(z\left(t_{n-1}+s\left(\tau-t_{n-1}\right)\right), t_{n-1}+s\left(\tau-t_{n-1}\right)\right) d s= \\
=\int_{t_{n-1}}^{t_{n}} d \tau \int_{0}^{1}\left[\left[\Phi^{\prime}\left(z\left(t_{n-1}+s\left(\tau-t_{n-1}\right)\right)\right) \dot{z}\left(t_{n-1}+s\left(\tau-t_{n-1}\right)\right)\left(\tau-t_{n-1}\right)+\right.\right. \\
\left.+\frac{\partial \Phi}{\partial t}\left(z\left(t_{n-1}+s\left(\tau-t_{n-1}\right)\right)\right)\left(\tau-t_{n-1}\right)\right] d \tau
\end{gathered}
$$

$(6.15)$

$$
=\int_{0}^{\omega_{n}} \theta d \theta \int_{0}^{1}\left[\Phi^{\prime}\left(z\left(t_{n-1}+s \theta\right), t_{n-1}+s \theta\right) \dot{z}\left(t_{n-1}+s \theta\right)+\frac{\partial \Phi}{\partial t}\left(z\left(t_{n-1}+s \theta\right), t_{n-1}+s \theta\right)\right] d s .
$$

Replacing $t_{n-1}+\theta$ by $\tau$ and using (6.1), one gets:

$$
\begin{aligned}
& \left.\left\|\psi_{n}\left(t_{n}\right)-z_{n}\right\| \leq \int_{0}^{\omega_{n}} d \tau \int_{t_{n-1}}^{t_{n}}\left(\| \Phi^{\prime}(z(s), s) \Phi(z(s), s)+\frac{\partial \Phi}{\partial t}(z(s), s)\right) \|\right) d s \leq \\
& \quad \leq \omega_{n} \int_{t_{n-1}}^{t_{n}}\left(\left\|\Phi^{\prime}(z(s), s)\right\|\|\Phi(z(s), s)\|+\left\|\frac{\partial \Phi}{\partial t}(z(s), s)\right\|\right) d s .
\end{aligned}
$$


From (5.19) and (6.4) one obtains:

$$
\begin{gathered}
\left\|\Phi^{\prime}(z(s), s)\right\|\|\Phi(z(s), s)\|+\left\|\frac{\partial \Phi}{\partial t}(z(s), s)\right\| \\
\leq\left(a_{3}(s)+a_{2}(s)\|\Phi(z(s), s)\|\right)\|\Phi(z(s), s)\| \\
+\beta_{1}(s)+b_{1}(s)\|\Phi(z(s), s)\|+b_{2}(s)\|\Phi(z(s), s)\|^{2} .
\end{gathered}
$$

(5.20) and the notation $\sigma_{1}=a_{2}+b_{2}$ from Theorem 5.3, one gets:

$$
\left\|\psi_{n}\left(t_{n}\right)-z_{n}\right\| \leq \omega_{n} \int_{t_{n-1}}^{t_{n}}\left(\frac{a_{3}(s)}{\mu_{1}(s)}+\frac{a_{2}(s)}{\mu_{1}^{2}(s)}+\beta_{1}(s)+\frac{b_{1}(s)}{\mu_{1}(s)}+\frac{b_{2}(s)}{\mu_{1}^{2}(s)}\right) d s .
$$

According to the assumptions of Theorem 5.3 conditions (4.5) of Theorem 4.2 are satisfied with $\gamma_{1}(t)=a_{1}(t)-b_{1}(t), \sigma_{1}(t), \beta_{1}(t)$ and $\mu_{1}(t)$ in place of $\gamma, \sigma, \beta$ and $\mu$ respectively. Thus one gets:

$$
\begin{gathered}
\left\|\psi_{n}\left(t_{n}\right)-z_{n}\right\| \leq \omega_{n} \int_{t_{n-1}}^{t_{n}}\left(\beta_{1}(s)+\frac{a_{3}(s)+b_{1}(s)}{\mu_{1}(s)}+\frac{a_{2}(s)+b_{2}(s)}{\mu_{1}^{2}(s)}\right) d s \\
\quad \leq \omega_{n} \int_{t_{n-1}}^{t_{n}}\left(\beta_{1}(s)+\frac{a_{1}(s)+a_{3}(s)-\gamma_{1}(s)}{\mu_{1}(s)}+\frac{\sigma_{1}(s)}{\mu_{1}^{2}(s)}\right) d s .
\end{gathered}
$$

Using inequalities (4.5), assumption (6.5), first assumption (6.6), the positivity and the monotonicity of $\mu_{1}(t)$, one gets the estimate:

$$
\begin{gathered}
\left\|\psi_{n}\left(t_{n}\right)-z_{n}\right\| \leq \omega_{n} \int_{t_{n-1}}^{t_{n}}\left(\frac{a_{1}(s)+a_{3}(s)}{\mu_{1}(s)}-\frac{\dot{\mu_{1}}}{\mu_{1}^{2}}\right) d s \\
\leq \omega_{n}\left(\frac{A \omega_{n}-1}{\mu_{1}\left(t_{n-1}\right)}+\frac{1}{\mu_{1}\left(t_{n}\right)}\right) \leq \omega_{n} \frac{1}{\mu_{1}\left(t_{n}\right)} .
\end{gathered}
$$

From (6.20) and (6.7) one gets:

$$
\left\|\psi_{n}\left(t_{n}\right)-z_{n}\right\|<\frac{\nu_{n}\left(\omega_{n}\right)}{\mu\left(t_{n}\right)} .
$$

From this inequality and (6.14) one obtains:

$$
\begin{gathered}
\left\|z_{n}-x\left(t_{n}\right)\right\| \leq\left\|z_{n}-\psi_{n}\left(t_{n}\right)\right\|+\left\|\psi_{n}\left(t_{n}\right)-x\left(t_{n}\right)\right\| \\
<\frac{\nu_{n}\left(\omega_{n}\right)}{\mu\left(t_{n}\right)}+\frac{1-\nu_{n}\left(\omega_{n}\right)}{\mu\left(t_{n}\right)}=\frac{1}{\mu\left(t_{n}\right)} .
\end{gathered}
$$

Thus (6.9) is proved.

Finally, (6.11) follows follows from (6.9) and (6.10). Theorem 6.1 is proved.

Remark 6.2. We prove now that a sequence $\left\{\omega_{n}\right\}$ which satisfies (6.6) and (6.7) does always exist. Choose an arbitrary $c \in(0,1)$ and consider a continuous function:

$$
\chi(s):=s-c \frac{\mu_{1}\left(t_{n-1}+s\right)}{\mu\left(t_{n-1}+s\right)} \nu_{n}(s)
$$


on the interval $[0,1 / A]$ with $\nu_{n}$ defined in (6.8). Clearly $\chi(0)<0$. Choose $\omega_{n}=1 / A$ if $\chi(s)<0$ on $[0,1 / A]$ and $\omega_{n}=s_{0}$ otherwise, where $s_{0}$ is the smallest zero of the function $\chi(s)$ on $[0,1 / A]$. Therefore

$$
\omega_{n}=\frac{1}{A} \quad \text { or } \quad \omega_{n}=c \frac{\mu_{1}\left(t_{n-1}+\omega_{n}\right)}{\mu\left(t_{n-1}+\omega_{n}\right)} \nu_{n}\left(\omega_{n}\right) .
$$

By (6.3) $t_{n}=t_{n-1}+\omega_{n}$. Since $0<c<1$, the constructed sequence $\left\{\omega_{n}\right\}$ satisfies the first condition in (6.6) and condition (6.7). To show that the second condition in (6.6) is also satisfied assume that

$$
\sum_{n=1}^{\infty} \omega_{n}=C<\infty
$$

Then (6.2) implies $t_{n} \leq C$ for all $n$. Therefore it follows from (6.24) that for every $n$ either $\omega_{n}=1 / A$ or

$$
\omega_{n}=c \frac{\mu_{1}\left(t_{n}\right)}{\mu\left(t_{n}\right)\left(\mu\left(t_{n}\right)\right.} \nu_{n}\left(\omega_{n}\right) \geq \tilde{c}>0 .
$$

Thus one has inequality $\omega_{n} \geq \min \{\tilde{c}, 1 / A\}>0$ for all $n$. This is a contradiction to (6.25).

\section{Regularized Continuous Methods For Monotone Operators}

In this section we apply the regularization procedure described in Sect. 3 to solve nonlinear operator equation (1.1) with a monotone operator $F$. Assume that $F(y)=0$ and $F$ is Fréchet differentiable in a ball $B_{2 r}(y)$.

Definition 7.1. A mapping $\varphi$ is monotone in a ball $B_{r}(y) \subset H$ if

$$
\left(\varphi\left(h_{1}\right)-\varphi\left(h_{2}\right), h_{1}-h_{2}\right) \geq 0, \quad \forall h_{1}, h_{2} \in B_{r}(y) .
$$

Note that a Fréchet differentiable operator is monotone in the ball $B_{2 r}(y)$ if and only if

$$
\left(F^{\prime}(h) \xi, \xi\right) \geq 0 \quad \text { for all } \quad h \in B_{2 r}(y), \quad \xi \in H .
$$

Definition 7.2. A mapping $\varphi$ is strongly monotone in a ball $B_{r}(y)$ if there exists a constant $k>0$ such that

$$
\left(\varphi\left(h_{1}\right)-\varphi\left(h_{2}\right), h_{1}-h_{2}\right) \geq k\left\|h_{1}-h_{2}\right\|^{2}, \quad \forall h_{1}, h_{2} \in B_{r}(y) .
$$

Under the assumption (7.1) the operator $F^{\prime}(h)+\varepsilon I$ is boundedly invertible for $h \in B_{2 r}(y)$ and for all positive $\varepsilon$. Define $\Phi$ as follows:

$$
\Phi(h, t):=-\left[F^{\prime}(h)+\varepsilon(t) I\right]^{-1}\left[F(h)+\varepsilon(t)\left(h-\tilde{z}_{0}\right)\right],
$$

where $\tilde{z}_{0}$ is a point belonging to $B_{r}(y)$, and $\varepsilon(t)$ is some positive function on the interval $[0, \infty)$. Some restrictions on $\varepsilon(t)$ will be stated in Theorem 7.12.

An outline of the convergence proof is the following. One considers an auxiliary well-posed problem:

$$
F_{\varepsilon}(x):=F(x)+\varepsilon(t)\left(x-\tilde{z}_{0}\right)=0, \quad \varepsilon>0,
$$

and shows that the difference between its solution $x(t)$ and the solution $z(t)$ to problem (5.5) tends to zero as $t \rightarrow+\infty$. On the other hand one shows that $x(t)$ converges to the exact solution $y$ of equation (1.1). Thus one proves the convergence of $z(t)$ to $y$ as $t \rightarrow+\infty$. 
We recall first some definitions from nonlinear functional analysis which are used below. The most essential restrictions on the operator $F$ imposed in this section are monotonicity and Fréchet differentiability in the ball $B_{2 r}(y)$.

Definition 7.3. A mapping $\varphi: H \rightarrow H$ is hemicontinuous if the map $t \rightarrow\left(\varphi\left(x_{0}+\right.\right.$ $\left.\left.t h_{1}\right), h_{2}\right)$ is continuous in a neighborhood of $t=0$ for any $x_{0}, h_{1}, h_{2} \in H$.

Definition 7.4. A mapping $\varphi: H \rightarrow H$ is coercive if $\|\varphi(h)\| \rightarrow \infty$ as $\|h\| \rightarrow \infty$.

Let $\rightarrow$ denote weak convergence and $\rightarrow$ denote strong convergence in $H$.

Definition 7.5. $\varphi$ is $w$-closed in a ball $B_{r}(y)$ if

$\left\{\left\{x_{n}\right\}_{n=1,2, \ldots} \subset B_{r}(y), x_{n} \rightarrow \xi\right.$ and $\left.\varphi\left(x_{n}\right) \rightarrow \eta\right\}$ implies $\varphi(\xi)=\eta$.

Lemma 7.6. If $\varphi$ is monotone and continuous in a ball $B_{r}(y)$, then $\varphi$ is $w$-closed in $B_{r}(y)$.

Proof of Lemma 7.6.

Consider a sequence $\left\{x_{n}\right\}_{n=1,2, \ldots} \subset B_{r}(y)$ such that $x_{n} \rightarrow \xi$ and $\varphi\left(x_{n}\right) \rightarrow \eta$. Take any $h \in H$ and sufficienly small positive $t$ such that $\xi+t h \in B_{r}(y)$. Since $\varphi$ is monotone in a ball $B_{r}(y)$, one has:

$$
\left(\varphi\left(x_{n}\right)-\varphi(\xi+t h), x_{n}-(\xi+t h)\right) \geq 0 .
$$

Since $\varphi\left(x_{n}\right) \rightarrow \eta$ and $x_{n} \rightarrow \xi$ one concludes $(\eta-\varphi(\xi+t h), h) \leq 0$. Let $t \rightarrow 0$ and use continuity of $\varphi$ to get $(\eta-\varphi(\xi), h) \leq 0$ for any $h \in H$. This implies $\eta=\varphi(\xi)$. Lemma is proved.

The well known result (see [14, p.100]) says that if an operator $\phi: H \rightarrow H$ is monotone, hemicontinuous, and coercive, then the equation $\phi(h)=0$ is uniquely solvable. Because the monotone operator $F$, which is studied in this section, is defined only in the ball $B_{2 r}(y)$, in the following Lemma the existence of the solution to equation (7.3) is proved for locally defined smooth monotone operators.

Lemma 7.7. If $F$ is monotone and Fréchet differentiable in a ball $B_{r}(y)$ then problem (7.3) is uniquely solvable in $B_{r}(y)$.

Proof of Lemma 7.7. Consider the Cauchy problem:

$$
\dot{z}=-F_{\varepsilon}(z), \quad z(0)=y,
$$

where $F_{\varepsilon}(z)=F(z)+\varepsilon\left(z-\tilde{z}_{0}\right), \varepsilon>0$ is a constant, and $\tilde{z}_{0} \in B_{r}(y)$. Then

$$
\begin{gathered}
\frac{1}{2} \frac{d}{d t}\left\|F_{\varepsilon}(z(t))\right\|^{2}=-\left(F_{\varepsilon}^{\prime}(z) F_{\varepsilon}(z), F_{\varepsilon}(z)\right) \\
=-\left(F^{\prime}(z) F_{\varepsilon}(z), F_{\varepsilon}(z)\right)-\varepsilon\left(F_{\varepsilon}(z), F_{\varepsilon}(z)\right) \leq-\varepsilon\left\|F_{\varepsilon}(z)\right\|^{2} .
\end{gathered}
$$

Therefore

$$
\frac{d}{d t}\left\|F_{\varepsilon}(z(t))\right\| \leq-\varepsilon\left\|F_{\varepsilon}(z(t))\right\|
$$

From this inequality one gets

$$
\left\|F_{\varepsilon}(z(t))\right\| \leq e^{-\varepsilon t}|| F_{\varepsilon}(y)\left\|\leq e^{-\varepsilon t} \varepsilon\right\| y-\tilde{z}_{0} \|<r \varepsilon e^{-\varepsilon t},
$$

if $z(t) \in B_{r}(y)$. Let us prove that $z(t) \in B_{r}(y)$ for all $t>0$ :

$$
\|z(t)-y\| \leq \int_{0}^{t}\left\|F_{\varepsilon}(z(s))\right\| d s \leq\left\|y-\tilde{z}_{0}\right\|\left(1-e^{-\varepsilon t}\right)<\left\|y-\tilde{z}_{0}\right\|<r .
$$


Hence $z(t)$ is defined for all $t \in[0,+\infty)$. Also

$$
\left\|z\left(t_{2}\right)-z\left(t_{1}\right)\right\| \leq \int_{t_{1}}^{t_{2}}\left\|F_{\varepsilon}(z(s))\right\| d s \leq r e^{-\varepsilon t_{1}} \rightarrow 0 \text { as } \min \left\{t_{1}, t_{2}\right\} \rightarrow+\infty .
$$

Thus, by the Cauchy criterion, there exists the strong limit: $x_{\varepsilon}:=\lim _{t \rightarrow \infty} z(t) \in$ $B_{r}(y)$. Since $F_{\varepsilon}$ is Fréchet differentiable in $B_{r}(y)$ it is continuous in $B_{r}(y)$ and $\lim _{t \rightarrow \infty}\left\|F_{\varepsilon}(z(t))\right\|=0$. Thus $x_{\varepsilon}$ is a solution to the equation (7.3) in $B_{r}(y)$.

Lemma 7.8. Suppose all the assumptions of Lemma 7.7 are satisfied, and $y$ is the unique solution to (1.1) in $B_{2 r}(y)$. Let $x(t)$ solve (7.3) for $\varepsilon=\varepsilon(t)$, and $\varepsilon(t)$ tend to zero as $t \rightarrow+\infty$. Then $x(t) \in B_{r}(y)$ for all $t \in[0,+\infty)$ and

$$
\lim _{t \rightarrow+\infty}\|x(t)-y\|=0 \text {. }
$$

\section{Proof of Lemma 7.8.}

First let us show that $x(t)$ is bounded. Indeed, it follows from (7.3) that

$$
F(x(t))-F(y)+\varepsilon(t)[x(t)-y]=\varepsilon(t)\left(\tilde{z}_{0}-y\right) .
$$

Therefore

$$
(F(x(t))-F(y), x(t)-y)+\varepsilon(t)\|x(t)-y\|^{2}=\varepsilon(t)\left(\tilde{z}_{0}-y, x(t)-y\right) .
$$

This and (7.1) imply

$$
\|x(t)-y\| \leq\left\|\tilde{z}_{0}-y\right\|<r,
$$

and therefore $F(x(t)) \rightarrow 0=F(y)$ as $t \rightarrow+\infty$.

Also, it follows from (7.8) that there exists a sequence $\left\{x\left(t_{n}\right)\right\}, t_{n} \rightarrow \infty$ as $n \rightarrow \infty$, which converges weakly to some element $\tilde{y} \in H$. Since $F$ is $w$-closed one gets that $F(\tilde{y})=0$, and, by the uniqueness of the solution to (1.1) in $B_{2 r}(y)$, it follows that $\tilde{y}=y$. Let us show that the sequence $\left\{x\left(t_{n}\right)\right\}$ converges strongly to $y$. Indeed, from (7.7), (7.1) and the relation $x\left(t_{n}\right) \rightarrow y$, one gets:

$$
\left\|x\left(t_{n}\right)-y\right\|^{2} \leq\left(\tilde{z}_{0}-y, x\left(t_{n}\right)-y\right) \rightarrow 0 \text { as } n \rightarrow \infty .
$$

Thus

$$
\lim _{n \rightarrow \infty}\left\|x\left(t_{n}\right)-y\right\|=0 .
$$

From (7.10) it follows by the standard argument that $x(t) \rightarrow y$ as $t \rightarrow \infty$. Lemma 7.8 is proved.

Lemma 7.9. Assume that $F$ is continuously Fréchet differentiable in a ball $B_{2 r}(y)$, $\sup _{x \in B_{2 r}(y)}\left\|F^{\prime}(x)\right\| \leq N_{1}$, and condition (7.1) holds. If $\varepsilon(t)$ is continuously differentiable, then the solution $x(t)$ to problem (7.3) with $\varepsilon=\varepsilon(t)$ is continuously differentiable in the strong sense and one has

$$
\|\dot{x}(t)\| \leq \frac{|\dot{\varepsilon}(t)|}{\varepsilon(t)}|| y-\tilde{z}_{0} \| \leq r \frac{|\dot{\varepsilon}(t)|}{\varepsilon(t)}, \quad t \in[0,+\infty) .
$$

\section{Proof of Lemma 7.9.}

Fréchet differentiability of $F$ implies hemicontinuity of $F$. Therefore problem (7.3) with $\varepsilon=\varepsilon(t)$ is uniquely solvable. The differentiability of $x(t)$ with respect to $t$ 
follows from the implicit function theorem ([14]). To derive (7.11) one differentiates equation (7.3) and uses the estimate $\left\|\left[F^{\prime}(x(t))+\varepsilon(t) I\right]^{-1}\right\| \leq \frac{1}{\varepsilon(t)}$. The result is:

$$
\|\dot{x}(t)\|=|\dot{\varepsilon}(t)| \cdot\left\|\left[F^{\prime}(x(t))+\varepsilon(t) I\right]^{-1}\left(x(t)-\tilde{z}_{0}\right)\right\| \leq \frac{|\dot{\varepsilon}(t)|}{\varepsilon(t)}\left\|y-\tilde{z}_{0}\right\| .
$$

Here we have used the estimate

$$
\left\|x(t)-\tilde{z}_{0}\right\| \leq\left\|y-\tilde{z}_{0}\right\|
$$

which can be derived from (7.3) similarly to the derivation of (7.8). Indeed,

$$
F(x(t))-F(y)+\varepsilon\left(x(t)-\tilde{z}_{0}\right)=0 .
$$

It follows from the monotonicity of $F$ that $\left(x(t)-\tilde{z}_{0}, x(t)-y\right) \leq 0$. Therefore

$$
\left(x(t)-\tilde{z}_{0}, x(t)-\tilde{z}_{0}\right) \leq\left(x(t)-\tilde{z}_{0}, y-\tilde{z}_{0}\right) \leq\left\|x(t)-\tilde{z}_{0}\right\| \cdot\left\|y-\tilde{z}_{0}\right\| .
$$

From this estimate and (7.8) one gets (7.13).

Finally, estimate (7.11) follows from (7.12) and (7.13).

Remark 7.10. Lemma 7.8 and formula (7.10) do not give a rate of convergence to $y$. This rate, in general, can be arbitrary slow.

To estimate $\|x(t)-y\|$ one may try to use the following inequality:

$$
\|x(t)-y\| \leq \int_{t}^{+\infty}\|\dot{x}(\tau)\| d \tau \leq r \int_{t}^{+\infty} \frac{|\dot{\varepsilon}(\tau)|}{\varepsilon(\tau)} d \tau .
$$

Since $\varepsilon(t) \rightarrow 0$ as $t \rightarrow+\infty$ monotonically, then $\int_{t}^{+\infty} \frac{|\dot{\varepsilon}(\tau)|}{\varepsilon(\tau)} d \tau=-\left.\log \varepsilon(t)\right|_{t} ^{\infty}=+\infty$, so in fact one can not use the above estimate in order to estimate the rate of convergence of $\|x(t)-y\|$.

This illustrates the strength of conclusion (7.6).

Lemma 7.11. Assume that $\varepsilon=\varepsilon(t)>0, F$ is twice Fréchet differentiable in $B_{2 r}(y)$, condition (7.1) holds, and

$$
\left\|F^{\prime}(x)\right\| \leq N_{1}, \quad\left\|F^{\prime \prime}(x)\right\| \leq N_{2} \quad \forall x \in B_{2 r}(y) .
$$

Then for the operator $\Phi$ defined by (7.2) and $x(t)$, the solution to (7.3) with $\varepsilon=\varepsilon(t)$, estimate (5.6) holds with

$$
\alpha(t) \equiv 0, \quad \gamma(t) \equiv 1, \text { and } \sigma(t):=\frac{N_{2}}{2 \varepsilon(t)} .
$$

\section{Proof of Lemma 7.11.}

Since $x(t)$ is the solution to (7.3) applying Taylor's formula one gets:

$$
\begin{aligned}
& (\Phi(h, t), h-x(t))=-\left(\left[F^{\prime}(h)+\varepsilon(t) I\right]^{-1}[F(h)-F(x(t))+\varepsilon(t)(h-x(t))], h-x(t)\right) \\
& \leq-\left(\left[F^{\prime}(h)+\varepsilon(t) I\right]^{-1}\left[F^{\prime}(h)(h-x(t))+\varepsilon(t)(h-x(t))\right], h-x(t)\right)+\frac{N_{2}\|h-x(t)\|^{3}}{2 \varepsilon(t)} \\
& =-\|h-x(t)\|^{2}+\frac{N_{2}\|h-x(t)\|^{3}}{2 \varepsilon(t)} .
\end{aligned}
$$

From (7.17) and (5.6) the conclusion of Lemma 7.11 follows.

Let us state the main result of this section. 
Theorem 7.12. Assume:

(1) problem (1.1) has a unique solution y in $B_{2 r}(y)$;

(2) $F$ is twice Fréchet differentiable in $B_{2 r}(y)$ and inequalities (7.1), (7.15) hold;

$$
z_{0}, \tilde{z}_{0} \in B_{r}(y)
$$

(4) $\varepsilon(t)>0$ is continuously differentiable, monotonically decreases to 0 as $t \rightarrow$ $+\infty$, and

$$
C_{\varepsilon}:=\max _{t \in[0, \infty)} \frac{\varepsilon(0)|\dot{\varepsilon}(t)|}{\varepsilon^{2}(t)}<1
$$

$$
\varepsilon(0)>\frac{N_{2}}{1-C_{\varepsilon}}\left\|z_{0}-x(0)\right\|
$$

$$
\varepsilon(0) \geq \frac{2 N_{2} C_{\varepsilon}}{\left(1-C_{\varepsilon}\right)^{2}}\left\|\tilde{z}_{0}-y\right\|
$$

$$
r \geq \frac{\varepsilon(0)\left(1-C_{\varepsilon}\right)}{N_{2}}
$$

(8) $\Phi$ is defined by (7.2).

Then the following conclusions hold:

i) Cauchy problem (5.5) has a unique solution $z(t) \subset B_{2 r}(y)$ for $t \in[0,+\infty)$, ii)

$$
\|z(t)-x(t)\| \leq \frac{1-C_{\varepsilon}}{N_{2}} \varepsilon(t), \quad \lim _{t \rightarrow+\infty}\|z(t)-y\|=0,
$$

iii)

$$
\begin{gathered}
\|F(z(t))\| \leq e^{-t}\left(\left\|F\left(z_{0}\right)\right\|+\varepsilon(0)\left\|\tilde{z}_{0}-z_{0}\right\|\right)+\frac{3 r}{1-C_{\varepsilon}} \varepsilon(t) \\
\leq\left(\frac{\left\|F\left(z_{0}\right)\right\|}{\varepsilon(0)}+\left\|\tilde{z}_{0}-z_{0}\right\|+\frac{3 r}{1-C_{\varepsilon}}\right) \varepsilon(t) .
\end{gathered}
$$

Remark 7.13. Note that Theorem 7.12 establishes convergence for any initial approximation point $z_{0}$ if $r$ is big enough and $\varepsilon(t)$ is appropriately chosen. To make an appropriate choice of $\varepsilon(t)$ one has to choose some function $\varepsilon(t)$ satisfying condition (7.19). Examples of such functions $\varepsilon(t)$ are given below. One can observe that condition (7.19) is invariant with respect to a multiplication $\varepsilon(t)$ by a constant. Therefore one can choose $\varepsilon(t)$ satisfying conditions (7.20) and (7.21) by a multiplication of the original $\varepsilon(t)$ by a sufficiently large constant. If $\frac{|\dot{\varepsilon}(t)|}{\varepsilon^{2}(t)}$ is not increasing, then in conditions (7.20) and (7.21) $C_{\varepsilon}:=\max _{t \in[0, \infty)} \frac{\varepsilon(0)|\dot{\varepsilon}(t)|}{\varepsilon^{2}(t)}$ can be replaced by $C_{\varepsilon}:=\frac{|\dot{\varepsilon}(0)|}{\varepsilon(0)}$. 
Remark 7.14. Inequalities (7.24) give the estimates for $\|F(z(t))\|$, the discrepancy of the continuous process. The first estimate in (7.24) shows that decay of $\|F(z(t))\|$ is estimated by the sum of two terms. The first term depends on $\|F(z(0))\|$ and on the distance between points $z_{0}$ and $\tilde{z}_{0}$. This term decreases with the rate $e^{-t}$ as in the well posed cases. The second term decreases slower because of the ill-posedness of the problem.

Remark 7.15. In order to get an estimate of the convergence rate for $\|x(t)-y\|$ one has to make some additional assumptions either on $F(x)$ or on the choice of the initial approximation $z_{0}$. Without such assumptions one cannot give an estimate of the convergence rate. Indeed, as a simple example consider the scalar equation $F(x):=x^{m}=0$. Then one gets the following algebraic equation for $x(\varepsilon)$ :

$$
F_{\varepsilon}(x):=x^{m}+\varepsilon\left(x-z_{0}\right)=0 .
$$

Assume $m$ is a positive integer and $z_{0}>0$. It is known that the solution to this equation is an algebraic function which can be represented by the Puiseux series: $x=\sum_{j=1}^{\infty} c_{j} \varepsilon^{\frac{j}{p}}$ in some neighborhood of zero. Thus $x=c_{1} \varepsilon^{\frac{1}{p}}(1+O(\varepsilon))$ as $\varepsilon \rightarrow 0$. Now from (7.25) one gets:

$$
c_{1}^{m} \varepsilon^{\frac{m}{p}}(1+O(\varepsilon))+c_{1} \varepsilon^{1+\frac{1}{p}}(1+O(\varepsilon))=z_{0} \varepsilon .
$$

Thus $p=m, c_{1}=z_{0}^{\frac{1}{m}}$ and $x(\varepsilon)=z_{0}^{\frac{1}{m}} \varepsilon^{\frac{1}{m}}(1+O(\varepsilon))$. For $\varepsilon=0$ one gets the solution $y=0$. Therefore

$$
|x(\varepsilon)-y| \sim \varepsilon^{\frac{1}{m}}, \quad \varepsilon \rightarrow 0,
$$

where $m$ can be chosen arbitrary large.

Below in Propositions 7.17 and 7.19 some sufficient conditions are given that allow one to obtain the estimates for $\|x(t)-y\|$.

Proof of Theorem 7.12.

For $\alpha(t), \gamma(t), \sigma(t)$ defined in (7.16), $\beta(t)$ defined in (5.7), and $v(t):=\| z(t)-$ $x(t) \|$ we are looking for a function $\mu(t)$ satisfying inequalities (4.5) and the second inequality in (4.6). Choose $\mu(t)=\frac{\lambda}{\varepsilon(t)}$, where $\lambda$ is a constant. Then rewrite first inequality (4.5) as

$$
N_{2} \leq \lambda\left(1-\frac{|\dot{\varepsilon}(t)|}{\varepsilon(t)}\right)
$$

Since $\alpha(t)=0$, using estimate (7.11) one gets:

$$
\beta(t)=\|\dot{x}(t)\| \leq \frac{|\dot{\varepsilon}(t)|}{\varepsilon(t)}\left\|y-\tilde{z}_{0}\right\| .
$$

Therefore the second inequality in (4.5) follows from the inequality:

$$
2|| \tilde{z}_{0}-y \| \frac{|\dot{\varepsilon}(t)|}{\varepsilon^{2}(t)} \leq \frac{1}{\lambda}\left(1-\frac{|\dot{\varepsilon}(t)|}{\varepsilon(t)}\right) .
$$

Also, the the second inequality in (4.6) can be rewritten as:

$$
\lambda\left\|z_{0}-x(0)\right\|<\varepsilon(0) .
$$

Choose

$$
\lambda:=\frac{N_{2}}{1-C_{\varepsilon}} .
$$


Then inequality (7.30) is the same as (7.20). It follows from (7.19) and the monotone decay of $\varepsilon(t)$ that

$$
\frac{|\dot{\varepsilon}(t)|}{\varepsilon(t)} \leq C_{\varepsilon} \frac{\varepsilon(t)}{\varepsilon(0)} \leq C_{\varepsilon} .
$$

From (7.31) and (7.32) one gets:

$$
\frac{N_{2}}{\lambda}=1-C_{\varepsilon} \leq 1-\frac{|\dot{\varepsilon}(t)|}{\varepsilon(t)},
$$

and (7.27) holds.

From (7.19), (7.21), and (7.31) one gets:

$$
\lambda=\frac{N_{2}}{1-C_{\varepsilon}} \leq \frac{\left(1-C_{\varepsilon}\right) \varepsilon(0)}{2 C_{\varepsilon}\left\|\tilde{z}_{0}-y\right\|} \leq \frac{1-\frac{\varepsilon(0)|\dot{\varepsilon}(t)|}{\varepsilon^{2}(t)}}{2\left\|\tilde{z}_{0}-y\right\| \mid \frac{\dot{\varepsilon}(t) \mid}{\varepsilon^{2}(t)}} .
$$

Hence one obtains:

$$
2|| \tilde{z}_{0}-y|| \frac{|\dot{\varepsilon}(t)|}{\varepsilon^{2}(t)} \leq \frac{1}{\lambda}\left(1-\frac{\varepsilon(0)|\dot{\varepsilon}(t)|}{\varepsilon^{2}(t)}\right) \leq \frac{1}{\lambda}\left(1-\frac{|\dot{\varepsilon}(t)|}{\varepsilon(t)}\right) .
$$

Therefore the first inequality in (7.29) also follows from the conditions of Theorem 7.12 .

It follows from the monotonicity of $\varepsilon(t)$ that for the chosen function $\mu(t)$ the assumption (7.22) of Theorem 7.12 is the same as the second inequality in (5.8). Thus all assumptions of Theorem 5.1 are satisfied. Applying Theorem 5.1 one concludes that $z(t) \in B_{2 r}(y)$ and inequality (7.21) holds. The second relation (7.23) follows from (7.6), inequality (7.23) and the triangle inequality:

$$
\|z(t)-y\| \leq\|z(t)-x(t)\|+\|x(t)-y\| .
$$

To prove (7.24), one uses (7.2) and gets:

$$
\left[F^{\prime}(z(t))+\varepsilon(t) I\right] \dot{z}(t)=-\left[F\left((z(t))+\varepsilon(t)\left(z(t)-\tilde{z}_{0}\right)\right] .\right.
$$

Denote:

$$
\rho(t):=\| F\left((z(t))+\varepsilon(t)\left(z(t)-\tilde{z}_{0}\right) \| .\right.
$$

Then it follows from (7.36) and (7.37) that

$$
\frac{d}{d t} \rho^{2}(t)=2\left(\left[F^{\prime}(z(t))+\varepsilon(t)\right] \dot{z}(t)+\varepsilon \dot{(t)}\left(z(t)-\tilde{z}_{0}\right), F\left((z(t))+\varepsilon(t)\left(z(t)-\tilde{z}_{0}\right)\right)\right.
$$

$$
\leq-2 \rho^{2}(t)+2 \rho(t)|\dot{\varepsilon}(t)|\left\|z(t)-\tilde{z}_{0}\right\| .
$$

Since $\rho(t)$ is a positive function, one obtains the following integral inequality:

$$
\frac{d}{d t} \rho(t) \leq-\rho(t)+|\dot{\varepsilon}(t)||| z(t)-\tilde{z}_{0} \| .
$$

It was already proved that $z(t) \in B_{2 r}(y)$. Therefore from assumption (7.18) one gets:

$$
\left\|z(t)-\tilde{z}_{0}\right\| \leq\|z(t)-y\|+\left\|\tilde{z}_{0}-y\right\| \leq 3 r .
$$

Using Lemma 4.1 one gets:

$$
\rho(t) \leq e^{-t} \rho(0)+3 r e^{-t} \int_{0}^{t} e^{s}|\dot{\varepsilon}(s)| d s
$$


Denote:

$$
g(t)=\int_{0}^{t} e^{s}|\dot{\varepsilon}(s)| d s, \quad f(t):=\frac{C_{\varepsilon}}{1-C_{\varepsilon}} e^{t} \varepsilon(t) .
$$

It follows from (7.32) that for $t \in[0, \infty)$ the following inequality holds:

$$
f^{\prime}(t)=\frac{C_{\varepsilon}}{1-C_{\varepsilon}} e^{t}[\varepsilon(t)+\dot{\varepsilon}(t)] \geq \frac{C_{\varepsilon}}{1-C_{\varepsilon}} e^{t}\left(\frac{|\dot{\varepsilon}(t)|}{C_{\varepsilon}}-|\dot{\varepsilon}(t)|\right)=e^{t}|\dot{\varepsilon}(t)|=g^{\prime}(t)
$$

Since $g(0)=0$, and by $(7.42) f(0)>0$, it follows that $f(0)>g(0)$. Thus $f(t)>g(t)$ for all $t \in[0, \infty)$ and one obtains the following inequality:

$$
e^{-t} \int_{0}^{t} e^{s}|\dot{\varepsilon}(s)| d s \leq \frac{C_{\varepsilon}}{1-C_{\varepsilon}} \varepsilon(t) .
$$

From (7.41) and (7.44) one obtains:

$$
\rho(t) \leq e^{-t} \rho(0)+3 r \frac{C_{\varepsilon}}{1-C_{\varepsilon}} \varepsilon(t) .
$$

It follows from (7.37) and the monotonicity of $\varepsilon(t)$ that

$$
\rho(0) \leq\left\|F\left(z_{0}\right)\right\|+\varepsilon(0)\left\|z_{0}-\tilde{z}_{0}\right\|,
$$

and

$$
\|F(z(t))\| \leq \rho(t)+\varepsilon(t)\left\|z(t)-\tilde{z}_{0}\right\| .
$$

Thus from (7.45) and (7.47) one gets

$$
\|F(z(t))\| \leq e^{-t}\left(\left\|F\left(z_{0}\right)\right\|+\varepsilon(0)\left\|z_{0}-\tilde{z}_{0}\right\|\right)+3 r \frac{C_{\varepsilon}}{1-C_{\varepsilon}} \varepsilon(t),
$$

and the first inequality in (7.24) is proved.

It follows from (7.32) that

$$
[\log (\varepsilon(t))]^{\prime} \geq\left[\log \left(\varepsilon(0) e^{-C_{\varepsilon} t}\right)\right]^{\prime} .
$$

Therefore:

$$
\log (\varepsilon(t)) \geq \log \left(\varepsilon(0) e^{-C_{\varepsilon} t}\right),
$$

and, since $C_{\varepsilon}<1$, one obtains:

$$
\varepsilon(t) \geq \varepsilon(0) e^{-t} .
$$

The second inequality in (7.24) follows from (7.51) and the first inequality (7.24). Theorem 7.12 is proved.

Example 7.16. 1. Let $\varepsilon(t)=\varepsilon_{0}\left(t_{0}+t\right)^{-\nu}, \varepsilon_{0}, t_{0}$ and $\nu$ are positive constants. Then $C_{\varepsilon}=\frac{\nu}{t_{0}}$ and condition (7.19) is satisfied if $\nu \in(0,1]$ and $t_{0}>\nu$.

2. If $\varepsilon(t)=\frac{\varepsilon_{0}}{\log \left(t_{0}+t\right)}$, then $C_{\varepsilon}=\frac{1}{t_{0} \log t_{0}}$ and condition (7.19) is satisfied if $t_{0} \log t_{0}>1$.

Note that if $\varepsilon(t)=\varepsilon_{0} e^{-\nu t}$ then condition (7.19) is not satisfied. 
Proposition 7.17. Let all the assumptions of Theorem 7.12 hold. Suppose also that the following inequality holds:

$$
(F(h), h-y) \geq c\|h-y\|^{1+a}, \quad a>0 .
$$

Then for the solution $z(t)$ to problem (5.5) with $\Phi$ defined in (7.2), the following estimate holds:

$$
\|z(t)-y\|=O\left(\varepsilon^{\min \left\{\frac{1}{a}, 1\right\}}(t)\right) .
$$

\section{Proof of Proposition 7.17.}

Denote $\|x(t)-y\|:=\varrho(t)$. Since $F(y)=0$, inequality (7.7) implies

$$
c \varrho^{1+a}(t)+\varepsilon(t) \varrho^{2}(t) \leq \varepsilon(t)\left\|\tilde{z}_{0}-y\right\| \varrho(t)
$$

and $\varrho(t) \rightarrow 0$ as $t \rightarrow+\infty$. This inequality can be reduced to

$$
c \varrho^{a}(t)+\varepsilon(t) \varrho(t) \leq \varepsilon(t)\left\|\tilde{z}_{0}-y\right\| .
$$

Thus $\varrho^{a}(t) \leq \frac{\left\|\tilde{z}_{0}-y\right\|}{c} \varepsilon(t)$, and

$$
\varrho(t)=\|x(t)-y\| \leq\left(\frac{\left\|\tilde{z}_{0}-y\right\|}{c}\right)^{\frac{1}{a}} \varepsilon^{\frac{1}{a}}(t) .
$$

Combining this estimate with estimate (7.23) for $\|z(t)-x(t)\|$ and using the triangle inequality one gets:

$$
\begin{gathered}
\|z(t)-y\| \leq\|z(t)-x(t)\|+\|x(t)-y\| \leq \\
\leq \frac{1-C_{\varepsilon}}{N_{2}} \varepsilon(t)+\left(\frac{\left\|\tilde{z}_{0}-y\right\|}{c}\right)^{\frac{1}{a}} \varepsilon^{\frac{1}{a}}(t) .
\end{gathered}
$$

Proposition 7.17 is proved.

Example 7.18. In the case of a scalar function $f(h)$ and even integer $a>0$ the estimate $f(h)(h-y) \geq c|h-y|^{1+a}$ means that $f(h)=(h-y)^{a} g(h)$, where $g(h) \geq$ $c>0$, and hence $y$ is a zero of multiplicity $a$ for $f$.

Proposition 7.19. Let all the assumptions of Theorem 7.12 hold and there exists $v \in H$ such that

$$
z_{0}-y=F^{\prime}(y) v, \quad\|v\|<\frac{2}{N_{2}} .
$$

Then the solution $z(t)$ to problem (5.5) satisfies the following convergence rate estimate:

$$
\|z(t)-y\| \leq\left[\frac{1-C_{\varepsilon}}{N_{2}}+\frac{4\|v\|}{2-N_{2}\|v\|}\right] \varepsilon(t)
$$

\section{Proof of Proposition 7.19.}

Note that $F(y)=0$. Therefore from (7.3) one gets:

$$
F(x(t))-F(y)+\varepsilon(t)(x-y)=\varepsilon(t)\left(z_{0}-y\right) .
$$

By the Lagrange formula one has:

$$
\left\{\int_{0}^{1}\left(F^{\prime}(y+s(x(t)-y)) d s+\varepsilon(t) I\right\}(x(t)-y)=\varepsilon(t)\left(z_{0}-y\right) .\right.
$$


Denote $Q_{\varepsilon}(x):=\int_{0}^{1}\left(F^{\prime}(y+s(x-y)) d s+\varepsilon I\right.$. From (7.60) it follows that

$\|x-y\|=\varepsilon\left\|Q_{\varepsilon}^{-1}(x) Q_{0}(y) v\right\| \leq \varepsilon\left\|Q_{\varepsilon}^{-1}(x)\left(Q_{0}(y)-Q_{\varepsilon}(x)\right) v\right\|+\varepsilon\left\|Q_{\varepsilon}^{-1}(x) Q_{\varepsilon}(x) v\right\|$.

Since $Q_{\varepsilon}(x)=Q_{0}(x)+\varepsilon I$, one obtains

$$
\begin{gathered}
\|x-y\| \leq \varepsilon\left\|Q_{\varepsilon}^{-1}(x)\left(Q_{0}(y)-Q_{0}(x)\right) v\right\|+\varepsilon\left\|Q_{\varepsilon}^{-1}(x) \varepsilon v\right\|+\varepsilon\|v\| \\
\leq \frac{N_{2}}{2}\|x-y\|\|v\|+2 \varepsilon\|v\| .
\end{gathered}
$$

Here we have used assumption (7.1) which implies the inequality $\left\|Q_{\varepsilon}^{-1}(x)\right\| \leq \frac{1}{\varepsilon}$.

From (7.61) and the inequality (7.58) one gets:

$$
\|x-y\| \leq \frac{4 \varepsilon\|v\|}{2-N_{2}\|v\|} .
$$

Using the triangle inequality from the first inequality (7.23), and inequalities (7.58) and (7.62), one gets:

$$
\|z(t)-y\| \leq\|z(t)-x(t)\|+\|x(t)-y\| \leq \frac{1-C_{\varepsilon}}{N_{2}} \varepsilon(t)+\frac{4\|v\|}{2-N_{2}\|v\|} \varepsilon(t) .
$$

For $\varepsilon=\varepsilon(t)$ and $x=x(t)$ satisfying the assumptions of Theorem 7.12 one concludes that estimate (7.59) holds.

Now we describe the simple iteration scheme for solving nonlinear equation (1.1). Define:

$$
\Phi(h, t):=-\left[F(h)+\varepsilon(t)\left(h-\tilde{z}_{0}\right)\right],
$$

where $\tilde{z}_{0} \in B_{r}(y)$ and $\varepsilon(t)>0$ is defined on $[0,+\infty)$. Some restrictions on $\varepsilon(t)$ will be stated in Theorem 7.22.

Lemma 7.20. Assume that $F$ is monotone, $\Phi$ is defined by (7.64), and $x(t)$ is a solution to problem (7.3) with $\varepsilon=\varepsilon(t)>0, t \in[0,+\infty)$. Then for $\gamma(t):=\varepsilon(t)>0$ and for $\sigma(t)=\alpha(t) \equiv 0$ inequality (5.6) holds.

Proof of Lemma 7.20.

Since $x(t)$ solves (7.3), by the monotonicity of $F$ one has:

$$
(\Phi(h, t), h-x(t))=-(F(h)-F(x(t)), h-x(t))-\varepsilon(t)(h-x(t), h-x(t))
$$

$$
\leq-\varepsilon(t)\|h-x(t)\|^{2} .
$$

Lemma 7.20 is proved.

Lemma 7.20 together with Lemma 7.21 presented below allow one to formulate the convergence result concerning the simple iteration procedure (see Theorem 7.22).

Lemma 7.21. Let $\nu(t)$ be locally integrable on $[0,+\infty)$. Suppose that there exists $T \geq 0$ such that $\nu(t) \in C^{1}[T,+\infty)$ and

$$
\nu(t)>0, \quad-\frac{\dot{\nu}(t)}{\nu^{2}(t)} \leq C, \quad \text { for } \quad t \in[T,+\infty) .
$$

Then

$$
\lim _{t \rightarrow+\infty} \int_{0}^{t} \nu(\tau) d \tau=+\infty
$$


Proof of Lemma 7.21.

One can integrate (7.66)

$$
-\int_{T}^{t} \frac{\dot{\nu}(\tau)}{\nu^{2}(\tau)} d \tau \leq \int_{T}^{t} C d \tau, \quad t \in[T,+\infty)
$$

and get

$$
\frac{1}{\nu(t)} \leq C(t-T)+\frac{1}{\nu(T)}
$$

Without loss of generality we can assume that $C>0$, and then

$$
\nu(t) \geq \frac{1}{C(t-T)+\frac{1}{\nu(T)}} .
$$

Integrating this inequality one gets (7.67) and completes the proof.

Lemmas $7.7-7.9$ and $7.20-7.21$ imply the following result.

Theorem 7.22. Assume that:

(1) problem (1.1) has a unique solution y in a ball $B_{2 r}(y)$;

(2) $F$ is monotone;

(3) $F$ is continuously Fréchet differentiable and

$$
\left\|F^{\prime}(h)\right\| \leq N_{1}, \quad \text { for all } h \in B_{2 r}(y) ;
$$

(4) $\varepsilon(t)>0$ is continuously differentiable, tends to zero monotonically as $t \rightarrow$ $+\infty$, and $\lim _{t \rightarrow+\infty} \frac{\dot{\varepsilon}(t)}{\varepsilon^{2}(t)}=0$.

Then, for $\Phi$ defined by (7.64), Cauchy problem (5.5) has a unique solution $z(t)$ for all $t \in[0,+\infty)$ and

$$
\lim _{t \rightarrow \infty}\|z(t)-y\|=0
$$

\section{Proof of Theorem 7.22.}

In order to verify the assumptions of Theorem 5.1 we use estimate (7.65) to conclude that $\alpha(t)=\sigma(t)=0$ and $\gamma(t)=\varepsilon(t)$ in formula (5.6). By $(5.7) \beta(t)=$ $\|\dot{x}(t)\|$ because $\alpha(t)=0$. By $(7.11)$

$$
\beta(t)=\|\dot{x}(t)\| \leq \frac{|\dot{\varepsilon}(t)|}{\varepsilon(t)}\left\|y-\tilde{z}_{0}\right\| .
$$

To apply Theorem 5.1 one has to find a function $\mu(t) \in C^{1}[0,+\infty)$ satisfying (4.5) and the second inequality in (4.6). This will be so if

$$
\frac{|\dot{\varepsilon}(t)|}{\varepsilon(t)}|| y-\tilde{z}_{0}\left\|\leq \frac{1}{2 \mu(t)}\left(\varepsilon(t)-\frac{\dot{\mu}(t)}{\mu(t)}\right), \quad \mu(0)\right\| x(0)-z_{0} \|<1 .
$$

The function $\mu(t)$ can be chosen as the solution to the differential equation

$$
-\frac{\dot{\mu}(t)}{\mu^{2}(t)}+\frac{\varepsilon(t)}{\mu(t)}=\frac{A|\dot{\varepsilon}(t)|}{\varepsilon(t)}
$$

where $A:=2\left\|y-\tilde{z}_{0}\right\|$. Denote $\rho(t):=\frac{1}{\mu(t)}$. Then

$$
\dot{\rho}(t)+\varepsilon(t) \rho(t)=\frac{A|\dot{\varepsilon}(t)|}{\varepsilon(t)} .
$$


Solving this equation, one gets:

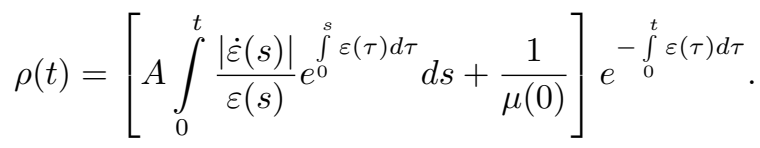

By Lemma $7.21 e^{\int^{t} \varepsilon(\tau) d \tau} \rightarrow \infty$ as $t \rightarrow \infty$. Applying L'Hôspital's rule to (7.71) and using condition 4 of Theorem 7.22 one gets:

$$
\lim _{t \rightarrow+\infty} \rho(t)=\lim _{t \rightarrow+\infty} \frac{|\dot{\varepsilon}(t)|}{\varepsilon^{2}(t)}=0 .
$$

Therefore $\mu(t)=1 / \rho(t)$ tends to $+\infty$ as $t \rightarrow+\infty$. To complete the proof one can take $\mu(0)$ sufficiently small for the second inequality in (7.69) to hold. By Theorem 5.1 one concludes that $\|z(t)-x(t)\| \rightarrow 0$ as $t \rightarrow+\infty$ and by Lemma 7.8 that $\|x(t)-y\| \rightarrow 0$ as $t \rightarrow+\infty$. Therefore it follows from the estimate:

$$
\|z(t)-y\| \leq\|z(t)-x(t)\|+\|x(t)-y\|,
$$

that $\|z(t)-y\| \rightarrow 0$ as $t \rightarrow+\infty$.

Remark 7.23. One has the estimate $\|z(t)-x(t)\| \leq \frac{1}{\mu(t)} \rightarrow 0$ as $t \rightarrow+\infty$. For the term $\|x(t)-y\|$ one can get the rate of convergence if some additional assumptions are made on $F$ or on $z_{0}$ (see Propositions 7.17, 7.19, and also Remark 7.15).

Remark 7.24. An interesting result similar to our Theorem 7.22 was established in [6, Theorem 8] for accretive operators in Banach space (in the case of Hilbert space accretive means monotone). The dynamical system considered in [6] is different from the one we study. In contrast to Theorem 8 in [6], where the existence of the global solution to the Cauchy problem for the corresponding nonlinear differential equation is one of the assumptions, we prove the existence and uniqueness of the solution to the corresponding Cauchy problem. The method of investigation in [6] is based on a linear differential inequality which is a particular case of (4.6) with $\sigma(t) \equiv 0$. This linear differential inequality has been used in the literature by many authors.

Example 7.25. 1. Let $\varepsilon(t)=\varepsilon_{0}(1+t)^{-\nu}, \varepsilon_{0}$ and $\nu$ are positive constants. Then the assumptions of Theorem 7.22 are satisfied if $\nu \in(0,1)$.

2. If $\varepsilon(t)=\frac{\varepsilon_{0}}{\log (1+t)}$, then the assumptions of Theorem 7.22 are satisfied.

If $\varepsilon(t)=\varepsilon_{0} e^{-\nu t}$ then condition 4 of Theorem 7.22 is not satisfied.

\section{Regularized Discrete Methods for Monotone Operators}

In this section we apply the results of Sections 6 and 7 to derive convergence theorems for regularized discrete methods.

First we consider the regularized Newton's method:

(8.1) $z_{n+1}=z_{n}-\omega_{n+1}\left[F^{\prime}\left(z_{n}\right)+\varepsilon\left(t_{n}\right) I\right]^{-1}\left[F\left(z_{n}\right)+\varepsilon\left(t_{n}\right)\left(z_{n}-\tilde{z}_{0}\right)\right], \quad n=0,1,2, \ldots$,

where $z_{0}, \tilde{z}_{0} \in B_{r}(y), t_{n+1}=t_{n}+\omega_{n+1}, t_{0}=0$.

Applying Theorem 6.1 to the regularized Newton's method one gets the following theorem.

Theorem 8.1. Assume:

(1) problem (1.1) has a unique solution y in $B_{2 r}(y)$; 
(2) $F$ is twice Fréchet differentiable in $B_{2 r}(y)$, and inequalities (7.1), (7.15) hold;

$$
z_{0}, \tilde{z}_{0} \in B_{r}(y)
$$

(4) $\varepsilon(t)>0$ is continuously differentiable and monotonically tends to 0 ,

$$
0<C_{\varepsilon}:=\max _{t \in[0, \infty)} \frac{\varepsilon(0)|\dot{\varepsilon}(t)|}{\varepsilon^{2}(t)} \leq \frac{1}{4}\left(2+\frac{N_{2}\left\|\tilde{z}_{0}-y\right\|}{\varepsilon(0)}\right)^{-1} ;
$$

$$
\begin{gathered}
\varepsilon(0)>\frac{N_{2}}{1-C_{\varepsilon}}\left\|z_{0}-x(0)\right\| ; \\
\varepsilon(0)>\frac{2 N_{2}}{1-2 C_{\varepsilon}}\left\|z_{0}-\tilde{z}_{0}\right\|+\sqrt{\frac{2 N_{2}}{1-2 C_{\varepsilon}}\left\|F\left(z_{0}\right)\right\| ;}
\end{gathered}
$$

(7) $\Phi$ is defined in (7.2),

$$
r \geq \frac{\varepsilon(0)\left(1-C_{\varepsilon}\right)}{N_{2}}
$$

$$
\omega_{n} \leq \frac{1}{2}, \quad \frac{\omega_{n}}{\nu_{n}\left(\omega_{n}\right)}<\frac{2\left(1-C_{\varepsilon}\right)}{1-2 C_{\varepsilon}}, \quad n=1,2, \ldots,
$$

where the continuous positive functions $\mu(t), \mu_{1}(t)$ are defined in Theorems 5.1 and 5.3, $\mu_{1}(t)$ is monotone, $\mu_{1}(t) \rightarrow \infty$ as $t \rightarrow+\infty$, and $\nu_{n}(t)$ is defined in (6.8).

Then the following conclusions hold:

i) all $z_{n}, n=1,2, \ldots$, defined in (8.1) belong to the ball $B_{2 r}(y)$,

ii)

$$
\left\|z_{n}-x\left(t_{n}\right)\right\| \leq \frac{1-C_{\varepsilon}}{N_{2}} \varepsilon\left(t_{n}\right)
$$

$$
t_{n} \rightarrow+\infty, \quad \varepsilon\left(t_{n}\right) \rightarrow 0 \quad \text { as } \quad n \rightarrow \infty
$$

iv)

$$
\lim _{n \rightarrow \infty}\left\|z_{n}-y\right\|=0
$$

\section{Proof of Theorem 8.1.}

Clearly the assumptions of Theorem 8.1 imply the assumptions of Theorem 7.12. Therefore the assumptions of Theorem 5.1 with $\mu(t)=\frac{\lambda}{\varepsilon(t)}, \lambda=\frac{N_{2}}{1-C_{\varepsilon}}$ are also satisfied (see the proof of Theorem 7.12). 
DYNAMICAL SYSTEMS AND DISCRETE METHODS FOR SOLVING NONLINEAR ILL-POSED PROBLEMBS

To check the assumptions of Theorem 5.3, consider $\Phi$ defined by formula (7.2). Since

$\Phi^{\prime}(h, t)=-\left[F^{\prime}(h)+\varepsilon(t) I\right]^{-1} F^{\prime \prime}(h)\left[F^{\prime}(h)+\varepsilon(t) I\right]^{-1}\left[F(h)+\varepsilon(t)\left(h-\tilde{z}_{0}\right)\right]-$

$$
-\left[F^{\prime}(h)+\varepsilon(t) I\right]^{-1}\left[F^{\prime}(h)+\varepsilon(t) I\right]
$$

for $h \in B_{2 r}(y)$ one gets:

$$
\begin{gathered}
\left(\Phi^{\prime}(h, t) \xi, \xi\right) \leq-\|\xi\|^{2}+\frac{\left\|F^{\prime \prime}(h)\right\|}{\varepsilon(t)}\|\Phi(h, t)\| \cdot\|\xi\|^{2} \\
\leq-\|\xi\|^{2}+\frac{N_{2}}{\varepsilon(t)}\|\Phi(h, t)\| \cdot\|\xi\|^{2} .
\end{gathered}
$$

Since $z(t) \in B_{2 r}(y)$ for any $t \in[0, \infty) \Phi^{\prime}$ satisfies condition (5.18) on $[0,+\infty)$ with

$$
a_{1}(t)=1, \quad a_{2}(t)=\frac{N_{2}}{\varepsilon(t)} .
$$

From (7.2) one has:

$$
\left[F^{\prime}(h)+\varepsilon(t) I\right] \Phi(h, t)=-F(h)-\varepsilon(t)\left(h-\tilde{z}_{0}\right) .
$$

Differentiating (8.15) with respect to $t$ one gets:

$$
\left[F^{\prime}(h)+\varepsilon(t) I\right] \frac{\partial \Phi}{\partial t}(h, t)=-\dot{\varepsilon}(t)\left[\Phi(h, t)+h-\tilde{z}_{0}\right] .
$$

Thus, with $h=z(t)$ in (8.16), using the estimate $\left\|F^{\prime}(h)+\varepsilon I\right\| \leq \frac{1}{\varepsilon}$ and the triangle inequality, one gets:

$$
\left\|\frac{\partial \Phi}{\partial t}(z(t), t)\right\| \leq \frac{|\dot{\varepsilon}(t)|}{\varepsilon(t)}\left(\|\Phi(z(t), t)\|+\|z(t)-x(t)\|+\left\|x(t)-\tilde{z}_{0}\right\|\right) .
$$

Using (7.13) and (7.23) one obtains:

$$
\left\|\frac{\partial \Phi}{\partial t}(z(t), t)\right\| \leq \frac{|\dot{\varepsilon}(t)|}{\varepsilon(t)}\left(\|\Phi(z(t), t)\|+\frac{1-C_{\varepsilon}}{N_{2}} \varepsilon(t)+\left\|\tilde{z}_{0}-y\right\|\right) .
$$

Since

$$
\frac{|\dot{\varepsilon}(t)|}{\varepsilon(t)} \leq C_{\varepsilon} \frac{\varepsilon(t)}{\varepsilon(0)} \quad \text { and } \quad 0<\frac{\varepsilon(t)}{\varepsilon(0)} \leq 1,
$$

condition (5.19) holds with

$$
\begin{gathered}
\beta_{1}(t)=\left(\frac{C_{\varepsilon}\left(1-C_{\varepsilon}\right)}{N_{2}}+\frac{C_{\varepsilon}\left\|\tilde{z}_{0}-y\right\|}{\varepsilon(0)}\right) \varepsilon(t), \\
b_{1}(t)=C_{\varepsilon} \frac{\varepsilon(t)}{\varepsilon(0)}, \quad \text { and } \quad b_{2}(t)=0 .
\end{gathered}
$$

Thus the functions $\gamma_{1}(t)$ and $\sigma_{1}(t)$ in Theorem 5.3 are:

$$
\begin{gathered}
\gamma_{1}(t):=a_{1}(t)-b_{1}(t)=1-C_{\varepsilon} \frac{\varepsilon(t)}{\varepsilon(0)}>\frac{1}{2}, \\
\sigma_{1}(t):=a_{2}(t)+b_{2}(t)=a_{2}(t)=\frac{N_{2}}{\varepsilon(t)},
\end{gathered}
$$


Choosing $\mu_{1}(t)=\frac{\lambda_{1}}{\varepsilon(t)}$, where $\lambda_{1}$ is a constant, one rewrites the assumptions of Theorem 5.3, that is, conditions (4.5) and the second inequality (4.6) with $v(0):=$ $v_{1}(0):=\left\|\Phi\left(z_{0}, 0\right)\right\|, \Phi$ defined in (8.3), and $t_{0}=0$, as:

$$
\begin{gathered}
N_{2} \leq \frac{\lambda_{1}}{2}\left(1-C_{\varepsilon} \frac{\varepsilon(t)}{\varepsilon(0)}-\frac{|\dot{\varepsilon}(t)|}{\varepsilon(t)}\right), \\
\frac{C_{\varepsilon}\left(1-C_{\varepsilon}\right)}{N_{2}}+\frac{C_{\varepsilon}\left\|\tilde{z}_{0}-y\right\|}{\varepsilon(0)} \leq \frac{1}{2 \lambda_{1}}\left(1-C_{\varepsilon} \frac{\varepsilon(t)}{\varepsilon(0)}-\frac{|\dot{\varepsilon}(t)|}{\varepsilon(t)}\right), \\
\lambda_{1}\left\|\left[F^{\prime}\left(z_{0}\right)+\varepsilon(0)\right]^{-1}\right\|\left\|F\left(z_{0}\right)+\varepsilon(0)\left(z_{0}-\tilde{z}_{0}\right)\right\|<\varepsilon(0) .
\end{gathered}
$$

Choose

$$
\lambda_{1}:=\frac{2 N_{2}}{1-2 C_{\varepsilon}} .
$$

From (8.3) one gets:

$$
\frac{|\dot{\varepsilon}(t)|}{\varepsilon(t)} \leq C_{\varepsilon} \frac{\varepsilon(t)}{\varepsilon(0)} \leq C_{\varepsilon} .
$$

Inequality (8.26) and definition (8.25) imply (8.22). From (8.26) and (8.3) one gets the following inequality:

$$
4 C_{\varepsilon}\left(1-C_{\varepsilon}\right)+4 \frac{C_{\varepsilon} N_{2}}{\varepsilon(0)}\left\|\tilde{z}_{0}-y\right\| \leq\left(1-2 C_{\varepsilon}\right)^{2} .
$$

Formula (8.23) is a consequence of (8.27).

Because of the monotonicity of $F$ one has:

$$
\left\|\left[F^{\prime}\left(z_{0}\right)+\varepsilon(0) I\right]^{-1}\right\| \leq \frac{1}{\varepsilon(0)} .
$$

Therefore inequality (8.24), with $\lambda_{1}$ as in (8.25), follows from the inequality:

$$
\varepsilon(0)^{2}-\frac{2 N_{2}\left\|\tilde{z}_{0}-z_{0}\right\|}{1-2 C_{\varepsilon}} \varepsilon(0)-\frac{2 N_{2}}{1-2 C_{\varepsilon}}\left\|F\left(z_{0}\right)\right\|>0 .
$$

Inequality (8.29) and therefore (8.24) is satisfied if

$$
\varepsilon(0)>\frac{N_{2}\left\|\tilde{z}_{0}-z_{0}\right\|}{1-2 C_{\varepsilon}}+\sqrt{\frac{N_{2}^{2}\left\|\tilde{z}_{0}-z_{0}\right\|^{2}}{\left(1-2 C_{\varepsilon}\right)^{2}}+\frac{2 N_{2}}{1-2 C_{\varepsilon}}\left\|F\left(z_{0}\right)\right\| .}
$$

This inequality is satisfied if (8.5) holds. Thus (8.5) implies (8.24). We have shown that the assumptions of Theorem 5.3 are satisfied.

Formula (8.12) implies assumption (6.4) of Theorem 6.1 with $a_{3}(t)=1$ and $a_{2}(t)$ defined in (8.14). Thus $A=2$ in (6.5). Therefore for $\mu(t)=\lambda / \varepsilon(t)$ and $\mu_{1}(t)=\lambda_{1} / \varepsilon(t)$ with $\lambda$ and $\lambda_{1}$ defined in (7.31) and (8.25), conditions (8.7) and (8.8) of Theorem 8.1 are the same as conditions (6.6) and (6.7) of Theorem 6.1. Hence one finishes the proof of Theorem 8.1 by applying Theorem 6.1.

Now consider a simple iteration method:

$$
z_{n+1}=z_{n}-\omega_{n+1}\left[F\left(z_{n}\right)+\varepsilon\left(t_{n}\right)\left(z_{n}-\tilde{z}_{0}\right)\right], \quad n=0,1,2, \ldots,
$$

where $z_{0}, \tilde{z}_{0} \in B_{r}(y), t_{n+1}=t_{n}+\omega_{n+1}, t_{0}=0$.

Theorem 8.2. Assume that:

(1) problem (1.1) has a unique solution $y$ in a ball $B_{2 r}(y)$; 
(2) F satisfies condition (7.1);

(3) $F$ is continuously Fréchet differentiable and

$$
\left\|F^{\prime}(h)\right\| \leq N_{1}, \quad \forall h \in B_{2 r}(y)
$$

(4) $\varepsilon(t)>0$ is continuously differentiable, tends to zero monotonically as $t \rightarrow$ $+\infty$, and $\lim _{t \rightarrow+\infty} \frac{\dot{\varepsilon}(t)}{\varepsilon^{2}(t)}=0 ;$

$$
\varepsilon(0)>C_{\varepsilon}
$$

where $C_{\varepsilon}$ is defined in (8.3);

$$
\frac{\left\|F\left(z_{0}\right)\right\|}{\varepsilon(0)}+\left\|z_{0}-\tilde{z}_{0}\right\|<\frac{6 r C_{\varepsilon}}{\varepsilon(0)-C_{\varepsilon}} ;
$$

(7) the sequence $\left\{z_{n}\right\}$ is defined in (8.31), $z_{0}, \tilde{z}_{0} \in B_{r}(y)$, and

$$
\sum_{n=1}^{\infty} \omega_{n}=\infty,
$$

$$
0<\omega_{n} \leq \frac{1}{N_{1}+2 \varepsilon(0)}, \quad \frac{\omega_{n}}{\nu_{n}\left(\omega_{n}\right)}<\frac{2\left(1-C_{\varepsilon}\right)}{1-2 C_{\varepsilon}}, \quad n=1,2, \ldots,
$$

where the continuous positive functions $\mu(t), \mu_{1}(t)$ are defined in Theorems 5.1 and 5.3, $\mu_{1}(t)$ is monotone, and $\nu_{n}(t)$ is defined in (6.8).

Then the following conclusions hold:

i) all $z_{n}, n=1,2, \ldots$, belong to the ball $B_{2 r}(y)$,

ii)

$$
\left\|z_{n}-x\left(t_{n}\right)\right\| \leq \frac{1-C_{\varepsilon}}{N_{2}} \varepsilon\left(t_{n}\right),
$$

iii)

$$
t_{n} \rightarrow+\infty, \quad \varepsilon\left(t_{n}\right) \rightarrow 0 \quad \text { as } \quad n \rightarrow \infty,
$$

$$
\lim _{n \rightarrow \infty}\left\|z_{n}-y\right\|=0
$$

\section{Proof of Theorem 8.2.}

The assumptions of Theorem 8.2 imply the assumptions of Theorem 7.22. Therefore the assumptions of Theorem 5.1 with $\mu(t)$ defined in (7.70) are also satisfied (see the proof of Theorem 7.22).

To check the assumptions of Theorem 5.3 consider $\Phi$ defined by formula (7.64). Since

$$
\Phi^{\prime}(h, t)=-F^{\prime}(h)-\varepsilon(t) I,
$$

condition (7.1) implies

$$
\left(\Phi^{\prime}(h, t) \xi, \xi\right)=-\left(F^{\prime}(h) \xi, \xi\right)-\varepsilon(t)\|\xi\|^{2} \leq-\varepsilon(t)\|\xi\|^{2}, \forall \xi \in H .
$$

Therefore $\Phi^{\prime}$ satisfies condition (5.18) with

$$
a_{1}(t)=\varepsilon(t), \quad \text { and } \quad a_{2}(t)=0 .
$$


For $h \in B_{2 r}(y)$ and $\tilde{z}_{0} \in B_{r}(y)$ one has:

$$
\left\|\frac{\partial \Phi}{\partial t}(z(t), t)\right\|=\left\|\dot{\varepsilon}(t)\left(h-\tilde{z}_{0}\right)\right\| \leq|\dot{\varepsilon}(t)|\left(\|h-y\|+\left\|\tilde{z}_{0}-y\right\|\right) \leq 3 r|\dot{\varepsilon}(t)| .
$$

Thus condition (5.19) also holds with

$$
\beta_{1}(t)=3 r|\dot{\varepsilon}(t)|, \quad b_{1}(t)=b_{2}(t)=0 .
$$

Since $\gamma_{1}(t)=\varepsilon(t), \sigma_{1}(t) \equiv 0$, and in Theorem $5.3 v(0)=\left\|\Phi\left(z_{0}, 0\right)\right\|=\| F\left(z_{0}\right)+$ $\varepsilon(0)\left(z_{0}-\tilde{z}_{0}\right) \|$ in order to satisfy the assumptions of Theorem 5.3 the function $\mu_{1}(t)$ should satisfy the following conditions:

$$
\begin{aligned}
& 3 r|\dot{\varepsilon}(t)| \leq \frac{1}{2 \mu_{1}(t)}\left(\varepsilon(t)-\frac{\dot{\mu}_{1}(t)}{\mu_{1}(t)}\right), \\
& \mu(0)\left\|F\left(z_{0}\right)+\varepsilon(0)\left(z_{0}-\tilde{z}_{0}\right)\right\|<1 .
\end{aligned}
$$

Choose

$$
\mu_{1}(t)=\frac{\lambda_{1}}{\varepsilon(t)}, \quad \lambda_{1}=\frac{\varepsilon(0)-C_{\varepsilon}}{6 r C_{\varepsilon}} .
$$

Hence assumptions (8.33) and (8.34) of Theorem 8.2 imply conditions (8.45) and (8.46). Therefore the assumptions of Theorem 5.3 are also satisfied.

From (8.40), (8.32) and the monotonicity of $\varepsilon(t)$ one concludes that assumption (6.4) of Theorem 6.1 holds with $a_{3}(t)=N_{1}+\varepsilon(t)$ and $a_{2}(t) \equiv 0$, and the assumption (6.5) with $A=N_{1}+2 \varepsilon(0)$.

To finish the proof of Theorem 8.2 one refers to Theorem 6.1.

\section{REFERENCES}

[1] Airapetyan, R.G. [2000] Continuous Newton method and its modification, Applicable Analysis, 73, N 3-4, pp. 463-484.

[2] Airapetyan, R.G. [2000] On new statement of inverse problem of Quantum Scattering Theory, Operator theory and its applications, Amer. Math. Soc., Providence RI, 2000, Fields Inst. Comm., 25.

[3] Airapetyan, R.G. and Puzynin, I.V. [1997] Newtonian iterative scheme with simultaneous iterations of inverse derivative, Comp. Phys. Comm., 102, pp. 97-108.

[4] Airapetyan, R.G., Ramm, A.G. and Smirnova, A.B. [1999] Continuous analog of GaussNewton method, Math. Models and Meth. in Appl. Sci., 9, N3, pp. 463-474.

[5] Airapetyan, R.G., Ramm, A.G. and Smirnova, A.B. [2000] Continuous methods for solving nonlinear ill-posed problems, Operator theory and its applications, Amer. Math. Soc., Providence RI, 2000, Fields Inst. Comm., 25, pp. 111-136.

[6] Alber, Ya.I. [1975] On a solution of operator equations of the first kind with accretive operators in Banach spaces, Diffferen. Uravneniya, 11, N12, 2242-2248.

[7] Alber, Ya.I. [1993] The regularization method for variational inequalities with nonsmooth unbounded operators in Banach space, Appl. Math. Lett., 6, N4, 63-68.

[8] Alber, Ya.I. [1994] A new approach to the investigation of evolution differential equations in Banach spaces, Nonlin. Anal., Theory, Methods \& Appl., 23, N9, 1115-1134.

[9] Argyros, I.K. [1998] Polynomial operator equations in abstract spaces and applications, CRC Press, Boca Raton.

[10] Beckenbach, E. and Bellman R. [1961] Inequalities, Springer-Verlag, Berlin.

[11] Blaschke, B., Neubauer, A. and Scherzer O. [1997] On convergence rates for the iteratively regularized Gauss-Newton method, IMA J. Num. Anal., 17, 421-436.

[12] Courant, R [1943] Variational methods for the solution of problems of equilibrium and vibrations, Bull. Amer. Math. Soc., 49, 1-23.

[13] Decker, D.W., Keller, H.B. and Kelley, C.T. [1983] Convergence rates for Newton's method at singular points, SIAM J. Numer. Anal., 20, N2, 296-314.

[14] Deimling, K. [1985] Nonlinear functional analysis, Springer-Verlag, New York. 
[15] Engl, H.W., Hanke, M. and Neubauer, A. [1996] Regularization of inverse problems, Kluwer Acad. Publ. Group, Dordrecht.

[16] Gavurin, M.K. [1958] Nonlinear functional equations and continuous analogies of iterative methods, Izv. Vuzov. Ser. Matematika. 5, pp. 18-31.

[17] Kamke, E. [1974] Differentialgleichungen. Lösungmethoden und Lösungen, Chelsea, New York.

[18] Kantorovich, L.V. and Akilov, G.P. [1982] Functional Analysis, Pergamon Press.

[19] Ortega, J.M. and Rheinboldt, W.C. [1970] Iterative Solution of Nonlinear Equations in Several Variables, Academic Press.

[20] Ramm, A.G. [1999] A numerical method for some nonlinear problems, Math. Models and Meth. in Appl.Sci., 9, N2, pp. 325-335.

[21] Ramm, A.G. and Smirnova, A.B. [1999] A numerical method for solving nonlinear ill-posed problems, Nonlinear Funct. Anal. and Optimiz., 20, N3, pp. 317-332.

[22] Ryazantseva, I.P. [1994] On some continuous regularization methods for monotone equations, Comput. Math. Math. Phys., 34, N1, 1-7.

[23] Szarski, J. [1967] Differential inequalities, PWN, Warszawa.

[24] Vasin, V.V. and Ageev, A.L., [1995] Ill-posed problems with a priori information, VNU, Utrecht.

[25] Zhidkov, E.P. and Puzynin, I.V. [1967] Solving of the boundary problems for second order nonlinear differential equations by means of the stabilization method, Soviet Math. Dokl. 8, pp. 614-616. 